\title{
Dual hesitant pythagorean fuzzy Hamacher aggregation operators in multiple attribute decision making
}

\author{
GUIWU WEI and MAO LU
}

\begin{abstract}
In this paper, we investigate the multiple attribute decision making (MADM) problem based on the Hamacher aggregation operators with dual Pythagorean hesitant fuzzy information. Then, motivated by the ideal of Hamacher operation, we have developed some Hamacher aggregation operators for aggregating dual hesitant Pythagorean fuzzy information. The prominent characteristic of these proposed operators are studied. Then, we have utilized these operators to develop some approaches to solve the dual hesitant Pythagorean fuzzy multiple attribute decision making problems. Finally, a practical example for supplier selection in supply chain management is given to verify the developed approach and to demonstrate its practicality and effectiveness.
\end{abstract}

Key words: multiple attribute decision making (MADM); dual Pythagorean hesitant fuzzy values; dual hesitant Pythagorean fuzzy Hamacher hybrid average (DHPFHHA) operator; dual hesitant Pythagorean fuzzy Hamacher hybrid geometric (DHPFHHG) operator; power aggregation operators.

\section{Introduction}

Atanassov [1,2] introduced the concept of intuitionistic fuzzy set (IFS) characterized by a membership function and a non-membership function, which is a generalization of the concept of fuzzy set [3] whose basic component is only a membership function. $\mathrm{Xu}$ [4] developed the intuitionistic fuzzy weighted averaging (IFWA) operator, intuitionistic fuzzy ordered weighted averaging (IFOWA) operator and the intuitionistic fuzzy hybrid aggregation (IFHA) operator. $\mathrm{Xu}$ [5] developed some geometric aggregation operators, such as the intuitionistic fuzzy weighted geometric (IFWG) operator, the intuitionistic fuzzy ordered weighted geometric (IFOWG) operator, and the intuitionistic fuzzy hybrid geometric (IFHG) operator and gave an application of the IFHG

The authors are with School of Business, Sichuan Normal University, Chengdu, 610101, P.R. China. The corresponding author is Guiwu Wei, e-mail: weiguiwu@163.com

This publication arises from research funded by the National Natural Science Foundation of China under Grant No. 61174149 and 71571128 and the Humanities and Social Sciences Foundation of Ministry of Education of the People's Republic of China (No. 16YJA630033) and the Construction Plan of Scientific Research Innovation Team for Colleges and Universities in Sichuan Province (15TD0004).

Received 4.3.2017. Revised 28.5.2017. 
operator to multiple attribute group decision making with intuitionistic fuzzy information. $\mathrm{Xu}$ and Yager [6] investigated the dynamic intuitionistic fuzzy multiple attribute decision making problems and developed some aggregation operators such as the dynamic intuitionistic fuzzy weighted averaging (DIFWA) operator and uncertain dynamic intuitionistic fuzzy weighted averaging (UDIFWA) operator to aggregate dynamic or uncertain dynamic intuitionistic fuzzy information. Wei [7] proposed some dynamic geometric aggregation operators such as the dynamic intuitionistic fuzzy weighted geometric (DIFWG) operator and uncertain dynamic intuitionistic fuzzy weighted geometric (UDIFWG) operator to aggregate dynamic or uncertain dynamic intuitionistic fuzzy information. Wei [8] proposed two new aggregation operators: induced intuitionistic fuzzy ordered weighted geometric (I-IFOWG) operator and induced interval-valued intuitionistic fuzzy ordered weighted geometric (I-IIFOWG) operator. Wei and Zhao [9] developed two new aggregation operators: induced intuitionistic fuzzy correlated averaging (I-IFCA) operator and induced intuitionistic fuzzy correlated geometric (I-IFCG) operator. Yu et al. [10] proposed some intuitionistic fuzzy aggregation operators such as the intuitionistic fuzzy prioritized weighted average (IFPWA) operator, the intuitionistic fuzzy prioritized weighted geometric (IFPWG) operator. $\mathrm{Xu}$ [11] developed a series of operators for aggregating intuitionistic fuzzy numbers and established various properties of these power aggregation operators. $\mathrm{Xu}$ and Chen [12] proposed an aggregation technique called the intuitionistic fuzzy Bonferroni mean for aggregating intuitionistic fuzzy information. Xu and Xia [13] studied the induced generalized aggregation operators under intuitionistic fuzzy environments. The intuitionistic fuzzy set has received more and more attention since its appearance[14-28].

More recently, Pythagorean fuzzy set (PFS) [29-30] has emerged as an effective tool for depicting uncertainty of the MADM problems. The PFS is also characterized by the membership degree and the non-membership degree, whose sum of squares is less than or equal to 1, the PFS is more general than the IFS. In some cases, the PFS can solve the problems that the IFS cannot, for example, if a DM gives the membership degree and the non-membership degree as 0.8 and 0.6 , respectively, then it is only valid for the PFS. In other words, all the intuitionistic fuzzy degrees are a part of the Pythagorean fuzzy degrees, which indicates that the PFS is more powerful to handle the uncertain problems. Zhang and $\mathrm{Xu}$ [31]provided the detailed mathematical expression for PFS and introduced the concept of Pythagorean fuzzy number (PFN). Meanwhile, they also developed a Pythagorean fuzzy TOPSIS (Technique for Order Preference by Similarity to Ideal Solution) for handling the MCDM problem within PFNs. Peng and Yang [32] proposed the division and subtraction operations for PFNs, and also developed a Pythagorean fuzzy superiority and inferiority ranking method to solve multicriteria group decision making problem with PFNs. Afterwards, Beliakov and James [33] focused on how the notion of "averaging" should be treated in the case of PFNs and how to ensure that the averaging aggregation functions produce outputs consistent with the case of ordinary fuzzy numbers. Reformat and Yager [34] applied the PFNs in handling the collaborative-based recommender system. 
In this paper, we introduce dual hesitant Pythagorean fuzzy set (DHPFS), which is a new extension of PFS and dual hesitant fuzzy set(DHFs) [35]. It's clear that the DHPFSs consist of two parts, that is, the membership degrees function and the non-membership degrees function, supporting a more exemplary and flexible access to assign values for each element in the domain, and we have to handle two kinds of degrees in this situation. For example, in a multiple attribute decision-making problem, some decision makers consider as possible values for the membership degree of into the set a few different values $0.4,0.5$, and 0.6 , and for the non-membership degrees $0.1,0.2$ and 0.3 replacing just one number or a tuple. So, the certainty and uncertainty on the possible values are somehow limited, respectively, which can reflect the original information given by the decision makers as much as possible. Utilizing DHPFSs can take much more information into account, the more values we obtain from the decision makers, the greater epistemic certainty we have, and thus, compared to the existing sets, DHPFS can be regarded as a more comprehensive set, which supports a more flexible approach when the decision makers provide their judgments.

Hamacher t-conorm and t-norm, which are the generalization of algebraic and Einstein t-conorm and t-norm [36], are more general and more flexible. There is important significance to research aggregation operators based on Hamacher operations and their application to MADM problems. However, all the above approaches are unsuitable to aggregate these dual hesitant Pythagorean fuzzy numbers on the basis of the Hamacher operations [37]. Thus, based on the Hamacher operations, how to aggregate these dual hesitant Pythagorean fuzzy numbers is an interesting topic. To solve this issue, in this paper, we shall develop some dual hesitant Pythagorean fuzzy Hamacher aggregation operators on the basis of the traditional Hamacher operations [37-42]. In order to do so, the remainder of this paper is set out as follows. In the next section, we introduce some basic concepts related to Pythagorean fuzzy set, dual hesitant Pythagorean fuzzy set and their operational laws. In Section 3, we shall propose some dual hesitant Pythagorean fuzzy Hamacher aggregation operators. In Section 4, we shall propose some dual hesitant Pythagorean fuzzy Hamacher power aggregation operators. In Section 5, based on these operators, we shall propose some models for multiple attribute decision making problems with dual hesitant Pythagorean fuzzy information. In Section 6, we present a numerical example for supplier selection in supply chain management with dual hesitant Pythagorean fuzzy information in order to illustrate the method proposed in this paper. Section 7 concludes the paper with some remarks.

\section{Preliminaries}

\subsection{Pythagorean fuzzy set}

The basic concepts of PFSs [29-30] are briefly reviewed in this section. Afterwards, novel score and accuracy functions for PFNs are proposed. Furthermore, a new comparison method for PFNs is developed. 
Definition 1 [29-30] Let X be a fix set. A PFS is an object having the form

$$
P=\left\{\left\langle x,\left(\mu_{P}(x), \nu_{P}(x)\right)\right\rangle \mid x \in X\right\}
$$

where $\mu_{P}: X \rightarrow[0,1]$ the function defines the degree of membership and the function $v_{P}:$ $X \rightarrow[0,1]$ defines the degree of non-membership of the element $x \in X$ to $P$, respectively, and, for every $x \in X$, it holds that

$$
\left(\mu_{p}(x)\right)^{2}+\left(v_{p}(x)\right)^{2} \leqslant 1 .
$$

Definition 2 [31] Let $\widetilde{a}_{1}=\left(\mu_{1}, v_{1}\right), \widetilde{a}_{2}=\left(\mu_{2}, v_{2}\right)$, and $\widetilde{a}=(\mu, v)$ be three Pythagorean fuzzy numbers, and some basic operations on them are defined as follows:

$$
\begin{aligned}
& \text { (1) } \widetilde{a}_{1} \oplus \widetilde{a}_{2}=\left(\sqrt{\left(\mu_{1}\right)^{2}+\left(\mu_{2}\right)^{2}-\left(\mu_{1}\right)^{2}\left(\mu_{2}\right)^{2}}, v_{1} v_{2}\right) ; \\
& \text { (2) } \widetilde{a}_{1} \otimes \widetilde{a}_{2}=\left(\mu_{1} \mu_{2}, \sqrt{\left(v_{1}\right)^{2}+\left(v_{2}\right)^{2}-\left(v_{1}\right)^{2}\left(v_{2}\right)^{2}}\right) \text {; } \\
& \text { (3) } \lambda \widetilde{a}=\left(\sqrt{1-\left(1-\mu^{2}\right)^{\lambda}, v^{\lambda}}\right), \lambda>0 ; \\
& \text { (4) }(\widetilde{a})^{\lambda}=\left(\mu^{\lambda} \sqrt{1-\left(1-v^{2}\right)^{\lambda}}\right), \lambda>0 ; \\
& \text { (5) } \widetilde{a}^{c}=(v, \mu) .
\end{aligned}
$$

\subsection{Dual hesitant Pythagorean fuzzy set}

In this section, we introduce dual hesitant Pythagorean fuzzy set (DHPFS), which is a new extension of PFS and dual hesitant fuzzy set [35]. It is clear that the DHPFSs consist of two parts, that is, the membership hesitancy function and the non-membership hesitancy function, supporting a more exemplary and flexible access to assign values for each element in the domain, and we have to handle two kinds of hesitancy in this situation.

Definition 3 Let $X$ be a fixed set, then a dual hesitant Pythagorean fuzzy set (DHPFS) on $X$ is described as:

$$
D=\left(\left\langle x, h_{P}(x), g_{P}(x)\right\rangle \mid x \in X\right)
$$

in which $h_{p}(x)$ and $g_{p}(x)$ are two sets of some values in $[0,1]$, denoting the possible membership degrees and non-membership degrees of the element $x \in X$ to the set $D$ respectively, with the conditions:

$$
\gamma^{2}+\eta^{2} \leqslant 1
$$

where $\gamma \in h_{P}(x), \eta \in g_{P}(x)$, for all $x \in X$. For convenience, the pair $d(x)=$ $\left(h_{P}(x), g_{P}(x)\right)$ is called a dual hesitant Pythagorean fuzzy number (DHPFN) denoted by $d=(h, g)$, with the conditions: $\gamma \in h, \eta \in g, 0 \leqslant \gamma, \eta \leqslant 1,0 \leqslant \gamma^{2}+\eta^{2} \leqslant 1$. 
To compare the DHPFNs, in the following, we shall give the following comparison laws:

Definition 4 Let $d=(h, g)$ be a DHPFNs, $s(d)=\frac{1}{2}\left(1+\frac{1}{\# h} \sum_{\gamma \in h} \gamma^{2}-\frac{1}{\# g} \sum_{\eta \in g} \eta^{2}\right)$ the score function of $d$, and $p(d)=\frac{1}{\# h} \sum_{\gamma \in h} \gamma^{2}+\frac{1}{\# g} \sum_{\eta \in g} \eta^{2}$ the accuracy function of $d$, where \#h and \#g are the numbers of the elements in $h$ and $g$ respectively, then, let $d_{i}=\left(h_{i}, g_{i}\right)(i=1,2)$ be any two DHPFNs, we have the following comparison laws:

- If $s\left(d_{1}\right)>s\left(d_{2}\right)$, then $d_{1}$ is superior to $d_{2}$, denoted by $d_{1} \succ d_{2}$;

- If $s\left(d_{1}\right)=s\left(d_{2}\right)$, then

(1) If $p\left(d_{1}\right)=p\left(d_{2}\right)$, then $d_{1}$ is equivalent to $d_{2}$, denoted by $d_{1} \sim d_{2}$;

(2) If $p\left(d_{1}\right)>p\left(d_{2}\right)$, then $d_{1}$ is superior to $d_{2}$, denoted by $d_{1} \succ d_{2}$.

Example 1 Let $d_{1}=\{\{0.3,0.4\},\{0.6\}\}, d_{2}=\{\{0.4,0.5\},\{0.3,0.4)\}$ by Definition 4, we can get:

$$
\begin{aligned}
& s\left(d_{1}\right)=\frac{1}{2}\left(1+\frac{1}{2}\left(0.3^{2}+0.4^{2}\right)-0.6^{2}\right)=0.3825 \\
& s\left(d_{2}\right)=\frac{1}{2}\left(1+\frac{1}{2}\left(0.4^{2}+0.5^{2}\right)-\frac{1}{2}\left(0.3^{2}+0.4^{2}\right)\right)=0.5400
\end{aligned}
$$

Thus, $s\left(d_{2}\right)>s\left(d_{1}\right)$, so $d_{2} \succ d_{1}$. Then, we define some new operations on the DHPFNs $d, d_{1}$ and $d_{2}$ :

(1) $d^{\lambda}=\cup_{\gamma \in h, \eta \in g}\left\{\left\{\gamma^{\lambda}\right\},\left\{\sqrt{1-\left(1-\eta^{2}\right)^{\lambda}}\right\}\right\}, \lambda>0$;

(2) $\lambda d=\cup_{\gamma \in h, \eta \in g}\left\{\left\{\sqrt{1-\left(1-\gamma^{2}\right)^{\lambda}}\right\},\left\{\eta^{\lambda}\right\}\right\}, \lambda>0$;

(3) $d_{1} \oplus d_{2}=\cup_{\gamma_{1} \in h_{1}, \gamma_{2} \in h_{2}, \eta_{1} \in g_{1}, \eta_{2} \in g_{2}}\left\{\left\{\sqrt{\left(\gamma_{1}\right)^{2}+\left(\gamma_{2}\right)^{2}-\left(\gamma_{1}\right)^{2}\left(\gamma_{2}\right)^{2}}\right\},\left\{\eta_{1} \eta_{2}\right\}\right\}$;

(4) $d_{1} \otimes d_{2}=\cup_{\gamma_{1} \in h_{1}, \gamma_{2} \in h_{2}, \eta_{1} \in g_{1}, \eta_{2} \in g_{2}}\left\{\left\{\gamma_{1} \gamma_{2}\right\},\left\{\sqrt{\left(\eta_{1}\right)^{2}+\left(\eta_{2}\right)^{2}-\left(\eta_{1}\right)^{2}\left(\eta_{2}\right)^{2}}\right\}\right\}$.

\subsection{Hamacher operations of dual hesitant Pythagorean fuzzy set}

Based on the traditional Hamacher operations [36], in the following, we shall define the Hamacher operations on the DHPFNs $d, d_{1}$ and $d_{2}$.

(1) $d^{\lambda}=\cup_{\gamma \in h, \eta \in g}\left\{\left\{\frac{\sqrt{\gamma}\left(\gamma_{1}\right)^{\lambda}}{\sqrt{\left(1+(\gamma-1)\left(1-\left(\gamma_{1}\right)^{2}\right)\right)^{\lambda}+(\gamma-1)\left(\gamma_{1}\right)^{2 \lambda}}}\right\}\right.$, 


$$
\begin{aligned}
& \left.\left\{\sqrt{\frac{\left(1+(\gamma-1)\left(\eta_{1}\right)^{2}\right)^{\lambda}-\left(1-\left(\eta_{1}\right)^{2}\right)^{\lambda}}{\left(1+(\gamma-1)\left(\eta_{1}\right)^{2}\right)^{\lambda}+(\gamma-1)\left(1-\left(\eta_{1}\right)^{2}\right)^{\lambda}}}\right\}\right\}, \lambda>0 ; \\
& \text { (2) } \lambda d=\cup_{\gamma \in h, \eta \in g}\left\{\left\{\sqrt{\frac{\left(1+(\gamma-1)\left(\gamma_{1}\right)^{2}\right)^{\lambda}-\left(1-\left(\gamma_{1}\right)^{2}\right)^{\lambda}}{\left(1+(\gamma-1)\left(\gamma_{1}\right)^{2}\right)^{\lambda}+(\gamma-1)\left(1-\left(\gamma_{1}\right)^{2}\right)^{\lambda}}}\right\}\right. \text {, } \\
& \left.\left\{\frac{\sqrt{\gamma}\left(\eta_{1}\right)^{\lambda}}{\sqrt{\left(1+(\gamma-1)\left(1-\left(\eta_{1}\right)^{2}\right)\right)^{\lambda}+(\gamma-1)\left(\eta_{1}\right)^{2 \lambda}}}\right\}\right\}, \lambda>0 \\
& \text { (3) } d_{1} \oplus d_{2}=\cup_{\gamma_{1} \in h_{1}, \gamma_{2} \in h_{2}, \eta_{1} \in g_{1}, \eta_{2} \in g_{2}}\left\{\left\{\sqrt{\frac{\left(\gamma_{1}\right)^{2}+\left(\gamma_{2}\right)^{2}-\left(\gamma_{1}\right)^{2}\left(\gamma_{2}\right)^{2}-(1-\gamma)\left(\gamma_{1}\right)^{2}\left(\gamma_{2}\right)^{2}}{1-(1-\gamma)\left(\gamma_{1}\right)^{2}\left(\gamma_{2}\right)^{2}}}\right\}\right. \text {, } \\
& \left.\left\{\frac{\eta_{1} \eta_{2}}{\sqrt{\gamma+(1-\gamma)\left(\left(\eta_{1}\right)^{2}+\left(\eta_{2}\right)^{2}-\left(\eta_{1}\right)^{2}\left(\eta_{2}\right)^{2}\right)}}\right\}\right\}, \lambda>0 \\
& \text { (4) } d_{1} \otimes d_{2}=\cup_{\gamma_{1} \in h_{1}, \gamma_{2} \in h_{2}, \eta_{1} \in g_{1}, \eta_{2} \in g_{2}}\left\{\left\{\frac{\gamma_{1} \gamma_{2}}{\sqrt{\gamma+(1-\gamma)\left(\left(\gamma_{1}\right)^{2}+\left(\gamma_{2}\right)^{2}-\left(\gamma_{1}\right)^{2}\left(\gamma_{2}\right)^{2}\right)}}\right\}\right. \text {, } \\
& \left.\left\{\sqrt{\frac{\left(\eta_{1}\right)^{2}+\left(\eta_{2}\right)^{2}-\left(\eta_{1}\right)^{2}\left(\eta_{2}\right)^{2}-(1-\gamma)\left(\eta_{1}\right)^{2}\left(\eta_{2}\right)^{2}}{1-(1-\gamma)\left(\eta_{1}\right)^{2}\left(\eta_{2}\right)^{2}}}\right\}\right\}, \lambda>0
\end{aligned}
$$

\section{Dual hesitant Pythagorean fuzzy Hamacher aggregation operators}

\subsection{Dual hesitant Pythagorean fuzzy Hamacher arithmetic aggregation operators}

In the following, we shall propose some dual hesitant Pythagorean fuzzy Hamacher arithmetic aggregation operator based on the Hamacher operations of DHPFNs.

Definition 5 Let $\widetilde{d}_{j}(j=1,2, \cdots, n)$ be a collection of DHPFNs, then we define the dual hesitant Pythagorean fuzzy Hamacher weighted average (DHPFHWA) operator as follows:

$$
\operatorname{DHPFHWA}\left(\widetilde{d}_{1}, \widetilde{d}_{2}, \cdots, \widetilde{d}_{n}\right)=\bigoplus_{j=1}^{n}\left(\omega_{j} \widetilde{d}_{j}\right)
$$

where $\omega=\left(\omega_{1}, \omega_{2}, \cdots, \omega_{n}\right)^{T}$ be the weight vector of $\widetilde{d}_{j}(j=1,2, \cdots, n)$, and $\omega_{j}>0$, $\sum_{j=1}^{n} \omega_{j}=1$.

Based on the operations of the dual hesitant Pythagorean fuzzy values described and mathematical induction method, we can drive the Theorem 1. 
Theorem 1 Let $\widetilde{d}_{j}(j=1,2, \cdots, n)$ be a collection of DHPFNs, then their aggregated value by using the DHPFHWA operator is also a DHPFN, and

$$
\begin{aligned}
& \text { DHPFHWA }\left(\widetilde{d}_{1}, \widetilde{d}_{2}, \cdots, \widetilde{d}_{n}\right) \\
& =\oplus_{j=1}^{n}\left(\omega_{j} \widetilde{d}_{j}\right) \\
& =\cup_{\gamma_{j} \in h_{j}, \eta_{j} \in g_{j}}\left\{\left\{\sqrt{\frac{\prod_{j=1}^{n}\left(1+(\gamma-1)\left(\gamma_{j}\right)^{2}\right)^{\omega_{j}}-\prod_{j=1}^{n}\left(1-\left(\gamma_{j}\right)^{2}\right)^{\omega_{j}}}{\prod_{j=1}^{n}\left(1+(\gamma-1)\left(\gamma_{j}\right)^{2}\right)^{\omega_{j}}+(\gamma-1) \prod_{j=1}^{n}\left(1-\left(\gamma_{j}\right)^{2}\right)^{\omega_{j}}}}\right\},\right. \\
& \left.\left\{\sqrt{\prod_{j=1}^{n} \prod_{j=1}^{n}\left(\eta_{j}\right)^{\omega_{j}}}\right\}\right\}
\end{aligned}
$$

where $\omega=\left(\omega_{1}, \omega_{2}, \cdots, \omega_{n}\right)^{T}$ be the weight vector of $\widetilde{d}_{j}(j=1,2, \cdots, n)$, and $\omega_{j}>0$, $\sum_{j=1}^{n} \omega_{j}=1, \gamma>0$.

Now, we can discuss some special cases of the DHPFHWA operator with respect to the parameter $\gamma$.

- When $\gamma=1$, DHPFHWA operator reduces to the dual hesitant Pythagorean fuzzy weighted average (DHPFWA) operator as follows:

$$
\begin{aligned}
& \operatorname{DHPFWA}_{\omega}\left(\widetilde{d}_{1}, \widetilde{d}_{2}, \cdots, \widetilde{d}_{n}\right) \\
& =\bigoplus_{j=1}^{n}\left(\omega_{j} \widetilde{d}_{j}\right) \\
& =\cup_{\gamma_{j} \in h_{j}, \eta_{j} \in g_{j}}\left\{\left\{\sqrt{1-\prod_{j=1}^{n}\left(1-\left(\gamma_{j}\right)^{2}\right)^{\omega_{j}}}\right\},\left\{\prod_{j=1}^{n}\left(\eta_{j}\right)^{\omega_{j}}\right\}\right\}
\end{aligned}
$$


- When $\gamma=2$, DHPFHWA operator reduces to the dual hesitant Pythagorean fuzzy Einstein weighted average (DHPFEWA) operator as follows:

$$
\begin{aligned}
& \operatorname{DHPFEWA}_{\omega}\left(\widetilde{d}_{1}, \widetilde{d}_{2}, \cdots, \widetilde{d}_{n}\right) \\
& =\bigoplus_{j=1}^{n}\left(\omega_{j} \widetilde{d}_{j}\right) \\
& =\cup_{\gamma_{j} \in h_{j}, \eta_{j} \in g_{j}}\left\{\left\{\sqrt{\frac{\prod_{j=1}^{n}\left(1+\left(\gamma_{j}\right)^{2}\right)^{\omega_{j}}-\prod_{j=1}^{n}\left(1-\left(\gamma_{j}\right)^{2}\right)^{\omega_{j}}}{\prod_{j=1}^{n}\left(1+\left(\gamma_{j}\right)^{2}\right)^{\omega_{j}}+\prod_{j=1}^{n}\left(1-\left(\gamma_{j}\right)^{2}\right)^{\omega_{j}}}}\right\},\right. \\
& \left.\left\{\frac{\sqrt{2} \prod_{j=1}^{n}\left(\eta_{j}\right)^{\omega_{j}}}{\sqrt{\prod_{j=1}^{n}\left(2-\left(\eta_{j}\right)^{2}\right)^{\omega_{j}}+\prod_{j=1}^{n}\left(\eta_{j}\right)^{2 \omega_{j}}}}\right\}\right\}
\end{aligned}
$$

Definition 6 Let $\widetilde{d}_{j}(j=1,2, \cdots, n)$ be a collection of DHPFNs, then we define the dual hesitant Pythagorean fuzzy Hamacher ordered weighted average (DHPFHOWA) operator as follows:

$$
\begin{aligned}
& \operatorname{DHPFHOWA}_{w}\left(\widetilde{d}_{1}, \widetilde{d}_{2}, \cdots, \widetilde{d}_{n}\right)=\bigoplus_{j=1}^{n}\left(w_{j} \widetilde{d}_{\sigma(j)}\right) \\
& =\cup_{\gamma_{\sigma(j)} \in h_{\sigma(j)}, \eta_{\sigma(j)} \in g_{\sigma(j)}}\left\{\sqrt{\frac{\prod_{j=1}^{n}\left(1+(\gamma-1)\left(\gamma_{\sigma(j)}\right)^{2}\right)^{w_{j}}-\prod_{j=1}^{n}\left(1-\left(\gamma_{\sigma(j)}\right)^{2}\right)^{w_{j}}}{\prod_{j=1}^{n}\left(1+(\gamma-1)\left(\gamma_{\sigma(j)}\right)^{2}\right)^{w_{j}}+(\gamma-1) \prod_{j=1}^{n}\left(1-\left(\gamma_{\sigma(j)}\right)^{2}\right)^{w_{j}}}}\right\}, \\
& \left.\left\{\frac{\sqrt{\gamma} \prod_{j=1}^{n}\left(\gamma_{\sigma(j)}\right)^{w_{j}}}{\sqrt{\prod_{j=1}^{n}\left(1+(\gamma-1)\left(1-\left(\gamma_{\sigma(j)}\right)^{2}\right)\right)^{w_{j}}+(\gamma-1) \prod_{j=1}^{n}\left(\gamma_{\sigma(j)}\right)^{2 w_{j}}}}\right\}\right\}
\end{aligned}
$$

where $(\sigma(1), \sigma(2), \cdots, \sigma(n))$ is a permutation of $1,2, \cdots, n$, such that $\widetilde{d}_{\sigma(j-1)} \geqslant \widetilde{d}_{\sigma(j)}$ for all $j=2, \cdots, n$, and $w=\left(w_{1}, w_{2}, \cdots, w_{n}\right)^{T}$ is the aggregation-associated weight vector such that $w_{j} \in[0,1]$ and $\sum_{j=1}^{n} w_{j}=1, \gamma>0$.

Now, we can discuss some special cases of the DHPFHOWA operator with respect to the parameter $\gamma$. 
- When $\gamma=1$, DHPFHOWA operator reduces to the dual hesitant Pythagorean fuzzy ordered weighted average (DHFOWA) operator as follows:

$$
\begin{gathered}
\operatorname{DHPFOWA}_{\mathrm{w}}\left(\widetilde{d}_{1}, \widetilde{d}_{2}, \cdots, \widetilde{d}_{n}\right)=\bigoplus_{j=1}^{n}\left(w_{j} \widetilde{d}_{\sigma(j)}\right) \\
=\cup_{\gamma_{\sigma(j)} \in h_{\sigma(j)}, \eta_{\sigma(j)} \in g_{\sigma(j)}}\left\{\left\{\sqrt{1-\prod_{j=1}^{n}\left(1-\left(\gamma_{\sigma(j)}\right)^{2}\right)^{w_{j}}}\right\},\right. \\
\left.\left\{\frac{\sqrt{2} \prod_{j=1}^{n} \gamma_{j}^{\omega_{j}}}{\sqrt{\prod_{j=1}^{n}\left(2-\left(\gamma_{j}\right)^{2}\right)^{\omega_{j}}+\prod_{j=1}^{n}\left(\gamma_{j}\right)^{2 \omega_{j}}}}\right\}\right\}
\end{gathered}
$$

- When $\gamma=2$, DHPFHOWA operator reduces to the dual hesitant Pythagorean fuzzy Einstein ordered weighted average (DHPFEOWA) operator as follows:

$$
\begin{aligned}
& \operatorname{DHPFEOWA}_{\mathrm{w}}\left(\widetilde{d}_{1}, \widetilde{d}_{2}, \cdots, \widetilde{d}_{n}\right)=\stackrel{\oplus}{j=1}^{n}\left(w_{j} \widetilde{d}_{\sigma(j)}\right) \\
& =\cup_{\gamma_{\sigma(j)} \in h_{\sigma(j)}, \eta_{\sigma(j)} \in g_{\sigma(j)}}\left\{\left\{\sqrt{\frac{\prod_{j=1}^{n}\left(1+\left(\gamma_{\sigma(j)}\right)^{2}\right)^{w_{j}}-\prod_{j=1}^{n}\left(1-\left(\gamma_{\sigma(j)}\right)^{2}\right)^{w_{j}}}{\prod_{j=1}^{n}\left(1+\left(\gamma_{\sigma(j)}\right)^{2}\right)^{w_{j}}+\prod_{j=1}^{n}\left(1-\left(\gamma_{\sigma(j)}\right)^{2}\right)^{w_{j}}}}\right\},\right. \\
& \left.\left.\qquad \frac{\sqrt{2} \prod_{j=1}^{n}\left(\eta_{\sigma(j)}\right)^{\omega_{j}}}{\sqrt{\prod_{j=1}^{n}\left(2-\left(\eta_{\sigma(j)}\right)^{2}\right)^{\omega_{j}}+\prod_{j=1}^{n}\left(\eta_{\sigma(j)}\right)^{2 \omega_{j}}}}\right\}\right\}
\end{aligned}
$$

From Definitions 5 and 6, we know that the DHPFHWA operator weights the dual hesitant Pythagorean fuzzy argument itself, while the DHPFHOWA operator weights the ordered positions of the dual hesitant Pythagorean fuzzy arguments instead of weighting the arguments themselves. Therefore, weights represent different aspects in both the DHPFHWA and DHPFHOWA operators. However, both the operators consider only one of them. To solve this drawback, in the following we shall propose a dual hesitant Pythagorean fuzzy Hamacher hybrid average (DHPFHHA) operator. 
Definition 7 A dual hesitant Pythagorean fuzzy Hamacher hybrid average (DHPFHHA) operator is defined as follows:

$$
\begin{aligned}
& \operatorname{DHPFHHA}_{w, \omega}\left(\tilde{d}_{1}, \tilde{d}_{2}, \cdots, \tilde{d}_{n}\right)=\bigoplus_{j=1}^{n}\left(w_{j} \dot{\tilde{d}}_{\sigma(j)}\right) \\
& =\cup_{\dot{\gamma}_{\sigma(j)} \in h_{\sigma(j)}, \dot{\eta}_{\sigma(j)} \in g_{\sigma(j)}}\left\{\sqrt{\frac{\prod_{j=1}^{n}\left(1+(\gamma-1)\left(\dot{\gamma}_{\sigma(j)}\right)^{2}\right)^{\omega_{j}}-\prod_{j=1}^{n}\left(1-\left(\dot{\gamma}_{\sigma(j)}\right)^{2}\right)^{\omega_{j}}}{\prod_{j=1}^{n}\left(1+(\gamma-1)\left(\dot{\gamma}_{\sigma(j)}\right)^{2}\right)^{\omega_{j}}+(\gamma-1) \prod_{j=1}^{n}\left(1-\left(\dot{\gamma}_{\sigma(j)}\right)^{2}\right)^{\omega_{j}}}}\right\}, \\
& \left.\left\{\frac{\sqrt{\gamma} \prod_{j=1}^{n}\left(\dot{\eta}_{\sigma(j)}\right)^{\omega_{j}}}{\sqrt{\prod_{j=1}^{n}\left(1+(\gamma-1)\left(1-\left(\dot{\eta}_{\sigma(j)}\right)^{2}\right)\right)^{\omega_{j}}+(\gamma-1) \prod_{j=1}^{n}\left(\dot{\eta}_{\sigma(j)}\right)^{2 \omega_{j}}}}\right\}\right\}
\end{aligned}
$$

where $w=\left(w_{1}, w_{2}, \cdots, w_{n}\right)$ is the associated weighting vector, with $w_{j} \in[0,1], \sum_{j=1}^{n} w_{j}=$ 1 , and $\dot{h}_{\sigma(j)}$ is the $j$-th largest element of the dual hesitant Pythagorean fuzzy arguments $\dot{\tilde{d}}\left(\dot{\widetilde{d}}=n \omega_{j} \widetilde{d}_{j}, j=1,2, \cdots, n\right), \omega=\left(\omega_{1}, \omega_{2}, \cdots, \omega_{n}\right)$ is the weighting vector of dual hesitant Pythagorean fuzzy arguments $\widetilde{d}_{j}(j=1,2, \cdots, n)$, with $\omega_{i} \in[0,1], \sum_{i=1}^{n} \omega_{i}=1$, and $n$ is the balancing coefficient, $\gamma>0$. Especially, if $w=(1 / n, 1 / n, \cdots, 1 / n)^{T}$, then $D H$ PFHA is reduced to the dual hesitant Pythagorean fuzzy weighted average (DHPFWA) operator; if, then DHPFHA is reduced to the dual hesitant Pythagorean fuzzy ordered weighted average (DHPFOWA) operator.

From Definition 7, we know that:

(1) The DHPFHHA operator first weights the given arguments, and then reorders the weighted arguments in descending order and weights these ordered arguments by the DHPFHHA weights, and finally aggregates all the weighted arguments into a collective one.

(2) The DHPFHHA operator generalizes both the DHPFHWA and DHPFHOWA operators, and reflects the importance degrees of both the given arguments and their ordered positions.

Now, we can discuss some special cases of the DHPFHHA operator with respect to the parameter $\gamma$. 
- When $\gamma=1$, DHPFHHA operator reduces to the hesitant Pythagorean fuzzy hybrid average (DHPFHA)operator as follows:

$$
\begin{aligned}
& \operatorname{DHPFHA}_{w, \omega}\left(\tilde{d}_{1}, \tilde{d}_{2}, \cdots, \tilde{d}_{n}\right)=\bigoplus_{j=1}^{n}\left(w_{j} \dot{\tilde{d}}_{\sigma(j)}\right) \\
& =\cup_{\dot{\gamma}_{\sigma(j)} \in h_{\sigma(j)}, \dot{\eta}_{\sigma(j)} \in g_{\sigma(j)}}\left\{\left\{\sqrt{1-\prod_{j=1}^{n}\left(1-\left(\dot{\gamma}_{\sigma(j)}\right)^{2}\right)^{w_{j}}}\right\},\left\{\prod_{j=1}^{n}\left(\dot{\eta}_{\sigma(j)}\right)^{w_{j}}\right\}\right\}
\end{aligned}
$$

- When $\gamma=2$, DHPFHHA operator reduces to the dual hesitant Pythagorean fuzzy Einstein hybrid average (HPFEHA) operator as follows:

$$
\left.\begin{array}{rl}
\operatorname{DHPFEHA}_{w, \omega} & \left(\widetilde{d}_{1}, \widetilde{d}_{2}, \cdots, \widetilde{d}_{n}\right)=\bigoplus_{j=1}^{n}\left(w_{j} \dot{\widetilde{d}}_{\sigma(j)}\right) \\
=\cup_{\dot{\gamma}_{\sigma(j)} \in h_{\sigma(j)}, \dot{\eta}_{\sigma(j)} \in g_{\sigma(j)}}\{ & \left\{\sqrt{\frac{\prod_{j=1}^{n}\left(1+\left(\dot{\gamma}_{\sigma(j)}\right)^{2}\right)^{w_{j}}-\prod_{j=1}^{n}\left(1-\left(\dot{\gamma}_{\sigma(j)}\right)^{2}\right)^{w_{j}}}{\prod_{j=1}^{n}\left(1+\left(\dot{\gamma}_{\sigma(j)}\right)^{2}\right)^{w_{j}}+\prod_{j=1}^{n}\left(1-\left(\dot{\gamma}_{\sigma(j)}\right)^{2}\right)^{w_{j}}}}\right\}, \\
& \left.\left\{\frac{\sqrt{2} \prod_{j=1}^{n}\left(\dot{\eta}_{\sigma(j)}\right)^{\omega_{j}}}{\sqrt{\prod_{j=1}^{n}\left(2-\left(\dot{\eta}_{\sigma(j)}\right)^{2}\right)^{\omega_{j}}+\prod_{j=1}^{n}\left(\dot{\eta}_{\sigma(j)}\right)^{2 \omega_{j}}}}\right\}\right\}
\end{array}\right\}
$$

\subsection{Dual hesitant Pythagorean fuzzy Hamacher Geometric Aggregation Operators}

Based on the dual hesitant Pythagorean fuzzy Hamacher arithmetic aggregation operators and the geometric mean, here we define some dual hesitant Pythagorean fuzzy Hamacher geometric aggregation operators:

Definition 8 Let $\widetilde{d}_{j}(j=1,2, \cdots, n)$ be a collection of DHPFNs, then we define the dual hesitant Pythagorean fuzzy Hamacher weighted geometric (DHPFHWG) operator as follows:

$$
\operatorname{DHPFHWG} \omega\left(\widetilde{d}_{1}, \widetilde{d}_{2}, \cdots, \widetilde{d}_{n}\right)=\otimes_{j=1}^{n}\left(\widetilde{d}_{j}\right)^{\omega_{j}}
$$

where $\omega=\left(\omega_{1}, \omega_{2}, \cdots, \omega_{n}\right)^{T}$ be the weight vector of $\widetilde{d}_{j}(j=1,2, \cdots, n)$, and $\omega_{j}>0$, $\sum_{j=1}^{n} \omega_{j}=1, \gamma>0$.

Based on the operations of the dual hesitant Pythagorean fuzzy values described and mathematical induction methods, we can drive the Theorem 2. 
Theorem 2 Let $\widetilde{d}_{j}(j=1,2, \cdots, n)$ be a collection of DHPFNs, then their aggregated value by using the DHPFHWG operator is also a DHPFN, and

$$
\begin{aligned}
& { }^{D H P F H W G} G_{\omega}\left(\widetilde{d}_{1}, \widetilde{d}_{2}, \cdots, \widetilde{d}_{n}\right) \\
& =\bigotimes_{j=1}^{n}\left(\widetilde{d}_{j}\right)^{\omega_{j}} \\
& =\cup_{\gamma_{j} \in h_{j}, \eta_{j} \in g_{j}}\left\{\left\{\frac{\sqrt{\gamma} \prod_{j=1}^{n} \gamma_{j}^{\omega_{j}}}{\sqrt{\prod_{j=1}^{n}\left(1+(\gamma-1)\left(1-\left(\gamma_{j}\right)^{2}\right)\right)^{\omega_{j}}+(\gamma-1) \prod_{j=1}^{n}\left(\gamma_{j}\right)^{2 \omega_{j}}}}\right\},\right. \\
& \left.\left\{\sqrt{\frac{\prod_{j=1}^{n}\left(1+(\gamma-1)\left(\eta_{j}\right)^{2}\right)^{\omega_{j}}-\prod_{j=1}^{n}\left(1-\left(\eta_{j}\right)^{2}\right)^{\omega_{j}}}{\prod_{j=1}^{n}\left(1+(\gamma-1)\left(\eta_{j}\right)^{2}\right)^{\omega_{j}}+(\gamma-1) \prod_{j=1}^{n}\left(1-\left(\eta_{j}\right)^{2}\right)^{\omega_{j}}}}\right\}\right\}
\end{aligned}
$$

where $\omega=\left(\omega_{1}, \omega_{2}, \cdots, \omega_{n}\right)^{T}$ be the weight vector of $\widetilde{d}_{j}(j=1,2, \cdots, n)$, and $\omega_{j}>0$, $\sum_{j=1}^{n} \omega_{j}=1, \gamma>0$.

Now, we can discuss some special cases of the DHPFHWG operator with respect to the parameter $\gamma$.

- When $\gamma=1$, DHPFHWG operator reduces to the dual hesitant Pythagorean fuzzy weighted geometric (DHPFWG) operator as follows:

$$
\begin{aligned}
& \operatorname{DHPFWG}_{\omega}\left(\widetilde{d}_{1}, \widetilde{d}_{2}, \cdots, \widetilde{d}_{n}\right)=\stackrel{\otimes}{\otimes}\left(\widetilde{d}_{j}\right)^{\omega_{j}} \\
& =\cup_{\gamma_{j} \in h_{j}, \eta_{j} \in g_{j}}\left\{\left\{\prod_{j=1}^{n}\left(\gamma_{j}\right)^{\omega_{j}}\right\},\left\{\sqrt{1-\prod_{j=1}^{n}\left(1-\left(\eta_{j}\right)^{2}\right)^{\omega_{j}}}\right\}\right\}
\end{aligned}
$$


- When $\gamma=2$, DHPFHWG operator reduces to the dual hesitant Pythagorean fuzzy Einstein weighted geometric (DHPFEWG) operator as follows:

$$
\begin{aligned}
& \operatorname{DHPFEWG}_{\omega}\left(\tilde{d}_{1}, \widetilde{d}_{2}, \cdots, \widetilde{d}_{n}\right)=\otimes_{j=1}^{n}\left(\widetilde{d}_{j}\right)^{\omega_{j}} \\
& =\cup_{\gamma_{j} \in h_{j}, \eta_{j} \in g_{j}}\left\{\left\{\frac{\sqrt{2} \prod_{j=1}^{n} \gamma_{j}^{\omega_{j}}}{\sqrt{\prod_{j=1}^{n}\left(2-\left(\gamma_{j}\right)^{2}\right)^{\omega_{j}}+\prod_{j=1}^{n}\left(\gamma_{j}\right)^{2 \omega_{j}}}}\right\}\right. \text {, } \\
& \left\{\sqrt{\frac{\prod_{j=1}^{n}\left(1+\left(\eta_{j}\right)^{2}\right)^{\omega_{j}}-\prod_{j=1}^{n}\left(1-\left(\eta_{j}\right)^{2}\right)^{\omega_{j}}}{\prod_{j=1}^{n}\left(1+\left(\eta_{j}\right)^{2}\right)^{\omega_{j}}+\prod_{j=1}^{n}\left(1-\left(\eta_{j}\right)^{2}\right)^{\omega_{j}}}}\right\}
\end{aligned}
$$

Definition 9 Let $\widetilde{d}_{j}(j=1,2, \cdots, n)$ be a collection of DHPFNs, then we define the dual hesitant Pythagorean fuzzy Hamacher ordered weighted geometric (DHPFHOWG?operator as follows:

$$
\begin{aligned}
& \operatorname{DHPFHOWG}_{w}\left(\widetilde{d}_{1}, \widetilde{d}_{2}, \cdots, \widetilde{d}_{n}\right)=\bigotimes_{j=1}^{n}\left(\widetilde{d}_{\sigma(j)}\right)^{w_{j}} \\
& =\cup_{\gamma_{\sigma(j)} \in h_{\sigma(j)}, \eta_{\sigma(j)} \in g_{\sigma(j)}}\left\{\left\{\frac{\sqrt{\gamma} \prod_{j=1}^{n}\left(\gamma_{\sigma(j)}\right)^{\omega_{j}}}{\sqrt{\prod_{j=1}^{n}\left(1+(\gamma-1)\left(1-\left(\gamma_{\sigma(j)}\right)^{2}\right)\right)^{\omega_{j}}+(\gamma-1) \prod_{j=1}^{n}\left(\gamma_{\sigma(j)}\right)^{2 \omega_{j}}}}\right\},\right. \\
& \left.\left\{\sqrt{\frac{\prod_{j=1}^{n}\left(1+(\gamma-1)\left(\eta_{\sigma(j)}\right)^{2}\right)^{w_{j}}-\prod_{j=1}^{n}\left(1-\left(\eta_{\sigma(j)}\right)^{2}\right)^{w_{j}}}{\prod_{j=1}^{n}\left(1+(\gamma-1)\left(\eta_{\sigma(j)}\right)^{2}\right)^{w_{j}}+(\gamma-1) \prod_{j=1}^{n}\left(1-\left(\eta_{\sigma(j)}\right)^{2}\right)^{w_{j}}}}\right\}\right\}
\end{aligned}
$$

where $(\sigma(1), \sigma(2), \cdots, \sigma(n))$ is a permutation of $(1,2, \cdots, n)$, such that $\widetilde{d}_{\sigma(j-1)} \geqslant \widetilde{d}_{\sigma(j)}$ for all $j=2, \cdots, n$, and $w=\left(w_{1}, w_{2}, \cdots, w_{n}\right)^{T}$ is the aggregation-associated weight vector such that $w_{j} \in[0,1]$ and $\sum_{j=1}^{n} w_{j}=1, \gamma>0$.

Now, we can discuss some special cases of the DHPFHOWG operator with respect to the parameter $\gamma$. 
- When $\gamma=1$, DHPFHOWG operator reduces to the dual hesitant Pythagorean fuzzy ordered weighted geometric (DHPFOWG) operator as follows:

$$
\begin{aligned}
& \operatorname{DHPFOWG}_{w}\left(\widetilde{d}_{1}, \widetilde{d}_{2}, \cdots, \widetilde{d}_{n}\right)=\stackrel{\bigotimes}{j=1}^{n}\left(\widetilde{d}_{\sigma(j)}\right)^{w_{j}} \\
& =\cup_{\gamma_{\sigma(j)} \in h_{\sigma(j)}, \eta_{\sigma(j)} \in g_{\sigma(j)}}\left\{\left\{\prod_{j=1}^{n}\left(\gamma_{\sigma(j)}\right)^{w_{j}}\right\},\left\{\sqrt{1-\prod_{j=1}^{n}\left(1-\left(\eta_{\sigma(j)}\right)^{2}\right)^{w_{j}}}\right\}\right\}
\end{aligned}
$$

- When $\gamma=2$, DHPFHOWG operator reduces to the dual hesitant Pythagorean fuzzy Einstein ordered weighted geometric (DHPFEOWG) operator as follows:

$$
\begin{aligned}
& \operatorname{DHPFEOWG}_{w}\left(\widetilde{d}_{1}, \widetilde{d}_{2}, \cdots, \widetilde{d}_{n}\right)=\bigotimes_{j=1}^{n}\left(\widetilde{d}_{\sigma(j)}\right)^{w_{j}} \\
& =\cup_{\gamma_{\sigma(j)} \in h_{\sigma(j)}, \eta_{\sigma(j)} \in g_{\sigma(j)}}\left\{\left\{\frac{\sqrt{2} \prod_{j=1}^{n}\left(\gamma_{\sigma(j)}\right)^{\omega_{j}}}{\sqrt{\prod_{j=1}^{n}\left(2-\left(\gamma_{\sigma(j)}\right)^{2}\right)^{\omega_{j}}+\prod_{j=1}^{n}\left(\gamma_{\sigma(j)}\right)^{2 \omega_{j}}}}\right\},\right. \\
& \left.\left\{\sqrt{\frac{\prod_{j=1}^{n}\left(1+\left(\eta_{\sigma(j)}\right)^{2}\right)^{w_{j}}-\prod_{j=1}^{n}\left(1-\left(\eta_{\sigma(j)}\right)^{2}\right)^{w_{j}}}{\prod_{j=1}^{n}\left(1+\left(\eta_{\sigma(j)}\right)^{2}\right)^{w_{j}}+\prod_{j=1}^{n}\left(1-\left(\eta_{\sigma(j)}\right)^{2}\right)^{w_{j}}}}\right\}\right\}
\end{aligned}
$$

From Definitions 8 and 9, we know that the DHPFHWG operator weights the dual hesitant Pythagorean fuzzy argument itself, while the DHPFHOWG operator weights the ordered positions of the dual hesitant Pythagorean fuzzy arguments instead of weighting the arguments themselves. Therefore, weights represent different aspects in both the DHPFHWG and DHPFHOWG operators. However, both the operators consider only one of them. To solve this drawback, in the following we shall propose a dual hesitant Pythagorean fuzzy Hamacher hybrid geometric (DHPFHHG) operator. 
Definition 10 A dual hesitant Pythagorean fuzzy Hamacher hybrid geometric (DHPFHHG) operator is defined as follows:

$$
\begin{aligned}
& \operatorname{DHPFHHG} G_{w, \omega}\left(\widetilde{d}_{1}, \widetilde{d}_{2}, \cdots, \widetilde{d}_{n}\right)=\bigotimes_{j=1}^{n}\left(\dot{\widetilde{d}}_{\sigma(j)}\right)^{w_{j}} \\
& =\cup_{\dot{\gamma}_{\sigma(j)} \in h_{\sigma(j)}, \dot{\eta}_{\sigma(j)} \in g_{\sigma(j)}}\left\{\left\{\frac{\sqrt{\gamma} \prod_{j=1}^{n}\left(\dot{\gamma}_{\sigma(j)}\right)^{\omega_{j}}}{\sqrt{\prod_{j=1}^{n}\left(1+(\gamma-1)\left(1-\left(\dot{\gamma}_{\sigma(j)}\right)^{2}\right)\right)^{\omega_{j}}+(\gamma-1) \prod_{j=1}^{n}\left(\dot{\gamma}_{\sigma(j)}\right)^{2 \omega_{j}}}}\right\},\right. \\
& \left.\left\{\sqrt{\frac{\prod_{j=1}^{n}\left(1+(\gamma-1)\left(\dot{\eta}_{\sigma(j)}\right)^{2}\right)^{\omega_{j}}-\prod_{j=1}^{n}\left(1-\left(\dot{\eta}_{\sigma(j)}\right)^{2}\right)^{\omega_{j}}}{\prod_{j=1}^{n}\left(1+(\gamma-1)\left(\dot{\eta}_{\sigma(j)}\right)^{2}\right)^{\omega_{j}}+(\gamma-1) \prod_{j=1}^{n}\left(1-\left(\dot{\eta}_{\sigma(j)}\right)^{2}\right)^{\omega_{j}}}}\right\}\right\}
\end{aligned}
$$

where $w=\left(w_{1}, w_{2}, \cdots, w_{n}\right)$ is the associated weighting vector, with $w_{j} \in[0,1], \sum_{j=1}^{n} w_{j}=$ 1 , and $\dot{h}_{\sigma(j)}$ is the $j$-th largest element of the dual hesitant Pythagorean fuzzy arguments $\dot{\tilde{d}}\left(\dot{\tilde{d}}=\left(\widetilde{d}_{j}\right)^{n \omega_{j}}, j=1,2, \cdots, n\right), \omega=\left(\omega_{1}, \omega_{2}, \cdots, \omega_{n}\right)$ is the weighting vector of dual hesitant Pythagorean fuzzy arguments $\tilde{d}_{j}(j=1,2, \cdots, n)$, with $\omega_{j} \in[0,1], \sum_{j=1}^{n} \omega_{j}=1$, and $n$ is the balancing coefficient, $\gamma>0$. Especially, if $w=(1 / n, 1 / n, \cdots, 1 / n)^{T}$, then DHPFHHG is reduced to the dual hesitant Pythagorean fuzzy weighted geometric ( $D H$ PFHWG) operator; if $\omega=(1 / n, 1 / n, \cdots, 1 / n)$, then DHPFHHG is reduced to the dual hesitant Pythagorean fuzzy ordered weighted geometric (DHPFHOWG) operator.

From Definition 10, we know that:

- When $\gamma=1$, DHPFHHG operator reduces to the dual hesitant Pythagorean fuzzy hybrid geometric (DHPFHG) operator as follows:

$$
\begin{aligned}
& \operatorname{DHPFHG}_{w, \omega}\left(\tilde{d}_{1}, \tilde{d}_{2}, \cdots, \tilde{d}_{n}\right)=\otimes_{j=1}^{n}\left(\dot{\tilde{d}}_{\sigma(j)}\right)^{w_{j}} \\
& =\cup_{\dot{\gamma}_{\sigma(j)} \in h_{\sigma(j)}, \dot{\eta}_{\sigma(j)} \in g_{\sigma(j)}}\left\{\left\{\prod_{j=1}^{n}\left(\dot{\gamma}_{\sigma(j)}\right)^{w_{j}}\right\},\left\{\sqrt{1-\prod_{j=1}^{n}\left(1-\left(\dot{\eta}_{\sigma(j)}\right)^{2}\right)^{w_{j}}}\right\}\right\}
\end{aligned}
$$


- When $\gamma=2$, DHPFHHG operator reduces to the dual hesitant Pythagorean fuzzy Einstein hybrid geometric (DHPFEHG) operator as follows:

$$
\begin{aligned}
& \operatorname{DHPFEHG}_{w, \omega}\left(\widetilde{d}_{1}, \widetilde{d}_{2}, \cdots, \widetilde{d}_{n}\right)=\bigotimes_{j=1}^{n}\left(\dot{\widetilde{d}}_{\sigma(j)}\right)^{w_{j}} \\
& =\cup_{\dot{\gamma}_{\sigma(j)} \in h_{\sigma(j)}, \dot{\eta}_{\sigma(j)} \in g_{\sigma(j)}}\left\{\left\{\frac{\sqrt{2} \prod_{j=1}^{n}\left(\dot{\gamma}_{\sigma(j)}\right)^{\omega_{j}}}{\sqrt{\prod_{j=1}^{n}\left(2-\left(\dot{\gamma}_{\sigma(j)}\right)^{2}\right)^{\omega_{j}}+\prod_{j=1}^{n}\left(\dot{\gamma}_{\sigma(j)}\right)^{2 \omega_{j}}}}\right\},\right. \\
& \left.\left\{\sqrt{\frac{\prod_{j=1}^{n}\left(1+\left(\dot{\eta}_{\sigma(j)}\right)^{2}\right)^{w_{j}}-\prod_{j=1}^{n}\left(1-\left(\dot{\eta}_{\sigma(j)}\right)^{2}\right)^{w_{j}}}{\prod_{j=1}^{n}\left(1+\left(\dot{\eta}_{\sigma(j)}\right)^{2}\right)^{w_{j}}+\prod_{j=1}^{n}\left(1-\left(\dot{\eta}_{\sigma(j)}\right)^{2}\right)^{w_{j}}}}\right\}\right\}
\end{aligned}
$$

\section{Dual hesitant Pythagorean fuzzy Hamacher power operators}

\subsection{Dual hesitant Pythagorean fuzzy Hamacher power Hamacher power weighted average (DHPFHPWA) operator}

Yager [43] developed a nonlinear weighted average aggregation operator called power average (PA) operator, which can be defined as follows:

$$
P A\left(a_{1}, a_{2}, \cdots, a_{n}\right)=\frac{\sum_{i=1}^{n}\left(1+T\left(a_{i}\right)\right) a_{i}}{\sum_{i=1}^{n}\left(1+T\left(a_{i}\right)\right)}
$$

where $T\left(a_{i}\right)=\sum_{\substack{j=1 \\ j \neq i}}^{n} \operatorname{Sup}\left(a_{i}, a_{j}\right)$, and $\operatorname{Sup}(a, b)$ is the support for $a$ from $b$, which satisfies the following three properties:

(1) $\operatorname{Sup}(a, b) \in[0,1]$;

(2) $\operatorname{Sup}(a, b)=\operatorname{Sup}(b, a)$;

(2) $\operatorname{Sup}(a, b) \geqslant \operatorname{Sup}(x, y)$.

In this section, we shall propose the dual hesitant Pythagorean fuzzy Hamacher power weighted average (DHPFHPWA) operator based on the power average [43] operators and Hamacher operations [36]. 
Definition 11 Let $\widetilde{d}_{j}(j=1,2, \cdots, n)$ be a collection of DHPFNs, then we define the dual hesitant Pythagorean fuzzy Hamacher power weighted average (DHPFHPWA) operator as follows:

$\operatorname{DHPFHPWA}\left(\widetilde{d}_{1}, \widetilde{d}_{2}, \cdots, \widetilde{d}_{n}\right)=\bigoplus_{j=1}^{n}\left(\frac{\omega_{j}\left(1+T\left(\tilde{d}_{j}\right)\right) \widetilde{d}_{j}}{\sum_{j=1}^{n} \omega_{j}\left(1+T\left(\tilde{d}_{j}\right)\right)}\right)=\cup_{\gamma_{j} \in h_{j}, \eta_{j} \in g_{j}}$

$\left\{\sqrt{\frac{\prod_{j=1}^{n}\left(1+(\gamma-1)\left(\gamma_{j}\right)^{2}\right)^{\frac{\omega_{j}\left(1+T\left(\tilde{d}_{j}\right)\right)}{\sum_{j=1}^{n} \omega_{j}\left(1+T\left(\widetilde{d}_{j}\right)\right)}}-\prod_{j=1}^{n}\left(1-\left(\gamma_{j}\right)^{2}\right)^{\frac{\omega_{j}\left(1+T\left(\tilde{d}_{j}\right)\right)}{\sum_{j=1}^{n} \omega_{j}\left(1+T\left(\widetilde{d}_{j}\right)\right)}}}{\prod_{j=1}^{n}\left(1+(\gamma-1)\left(\gamma_{j}\right)^{2}\right)^{\frac{\omega_{j}\left(1+T\left(\tilde{d}_{j}\right)\right)}{\sum_{j=1}^{n} \omega_{j}\left(1+T\left(\widetilde{d}_{j}\right)\right)}}+(\gamma-1) \prod_{j=1}^{n}\left(1-\left(\gamma_{j}\right)^{2}\right)^{\frac{\omega_{j}\left(1+T\left(\tilde{d}_{j}\right)\right)}{\sum_{j=1}^{n} \omega_{j}\left(1+T\left(\tilde{d}_{j}\right)\right)}}}}\right\}$,

$\left.\left\{\frac{\sqrt{\gamma} \prod_{j=1}^{n}\left(\eta_{j}\right)^{\frac{\omega_{j}\left(1+T\left(\widetilde{d}_{j}\right)\right)}{\sum_{j=1}^{n} \omega_{j}\left(1+T\left(\tilde{d}_{j}\right)\right)}}}{\sqrt{\prod_{j=1}^{n}\left(1+(\gamma-1)\left(1-\left(\eta_{j}\right)^{2}\right)\right)^{\frac{\omega_{j}\left(1+T\left(\tilde{d}_{j}\right)\right)}{\sum_{j=1}^{n} \omega_{j}\left(1+T\left(\widetilde{d}_{j}\right)\right)}}+(\gamma-1) \prod_{j=1}^{n}\left(\eta_{j}\right)^{\frac{2 \omega_{j}\left(1+T\left(\tilde{d}_{j}\right)\right)}{\sum_{j=1}^{n} \omega_{j}\left(1+T\left(\tilde{d}_{j}\right)\right)}}}}\right\}\right\}$

where $\omega=\left(\omega_{1}, \omega_{2}, \cdots, \omega_{n}\right)^{T}$ be the weight vector of $\widetilde{d}_{j}(j=1,2, \cdots, n), \gamma>0$, and

$$
T\left(\widetilde{d}_{j}\right)=\sum_{\substack{i=1 \\ i \neq j}}^{n} \omega_{i} \operatorname{Sup}\left(\widetilde{d}_{j}, \widetilde{d}_{i}\right)
$$

and $\operatorname{Sup}\left(\widetilde{d}_{j}, \widetilde{d}_{i}\right)$ is the support for $\widetilde{d}_{j}$ from $\widetilde{d}_{i}$, with the conditions:

- $\operatorname{Sup}\left(\widetilde{d}_{i}, \widetilde{d}_{j}\right) \in[0,1]$;

- $\operatorname{Sup}\left(\widetilde{d}_{i}, \widetilde{d}_{j}\right)=\operatorname{Sup}\left(\widetilde{d}_{i}, \widetilde{d}_{j}\right)$;

- $\operatorname{Sup}\left(\widetilde{d}_{i}, \widetilde{d}_{j}\right) \geqslant \operatorname{Sup}\left(\widetilde{d}_{s}, \widetilde{d}_{t}\right)$, if dis $\left(\widetilde{d}_{i}, \widetilde{d}_{j}\right) \geqslant \operatorname{dis}\left(\widetilde{d}_{s}, \widetilde{d}_{t}\right)$, where dis is a distance measure.

Now, we can discuss some special cases of the DHPFHWA operator with respect to the parameter $\gamma$. 
- When $\gamma=1$, DHPFHPWA operator reduces to the dual hesitant Pythagorean fuzzy power weighted average (DHPFPWA) operator as follows:

$$
\begin{aligned}
& \operatorname{DHPFPWAA}_{\omega}\left(\widetilde{d}_{1}, \widetilde{d}_{2}, \cdots, \widetilde{d}_{n}\right) \\
& =\bigoplus_{j=1}^{n}\left(\frac{\omega_{j}\left(1+T\left(\widetilde{d}_{j}\right)\right) \widetilde{d}_{j}}{\sum_{j=1}^{n} \omega_{j}\left(1+T\left(\widetilde{d}_{j}\right)\right)}\right) \\
& =\cup_{\gamma_{j} \in h_{j}, \eta_{j} \in g_{j}}\left\{\sqrt{1-\prod_{j=1}^{n}\left(1-\left(\gamma_{j}\right)^{2}\right)^{\omega_{j}\left(1+T\left(\widetilde{d}_{j}\right)\right) / \sum_{j=1}^{n} \omega_{j}\left(1+T\left(\widetilde{d}_{j}\right)\right)}}\right\}, \\
& \left.\left\{\prod_{j=1}^{n}\left(\eta_{j}\right)^{\omega_{j}\left(1+T\left(\widetilde{d}_{j}\right)\right) / \sum_{j=1}^{n} \omega_{j}\left(1+T\left(\widetilde{d}_{j}\right)\right)}\right\}\right\}
\end{aligned}
$$

- When $\gamma=2$, DHPFHPWA operator reduces to the dual hesitant Pythagorean fuzzy Einstein power weighted average (DHPFEPWA) operator as follows:

$$
\begin{aligned}
& \operatorname{DHPFEPWA}_{\omega}\left(\widetilde{d}_{1}, \widetilde{d}_{2}, \cdots, \widetilde{d}_{n}\right)=\bigoplus_{j=1}^{n}\left(\frac{\omega_{j}\left(1+T\left(\widetilde{d}_{j}\right)\right) \widetilde{d}_{j}}{\sum_{j=1}^{n} \omega_{j}\left(1+T\left(\widetilde{d}_{j}\right)\right)}\right)=\cup_{\gamma_{j} \in h_{j}, \eta_{j} \in g_{j}} \\
& \left\{\sqrt{\frac{\prod_{j=1}^{n}\left(1+\left(\gamma_{j}\right)^{2}\right)^{\frac{\omega_{j}\left(1+T\left(\tilde{d}_{j}\right)\right)}{\sum_{j=1}^{n} \omega_{j}\left(1+T\left(\widetilde{d}_{j}\right)\right)}}-\prod_{j=1}^{n}\left(1-\left(\gamma_{j}\right)^{2}\right)^{\frac{\omega_{j}\left(1+T\left(\widetilde{d}_{j}\right)\right)}{\sum_{j=1}^{n} \omega_{j}\left(1+T\left(\widetilde{d}_{j}\right)\right)}}}{\prod_{j=1}^{n}\left(1+\left(\gamma_{j}\right)^{2}\right)^{\frac{\omega_{j}\left(1+T\left(\tilde{d}_{j}\right)\right)}{\sum_{j=1}^{n} \omega_{j}\left(1+T\left(\widetilde{d}_{j}\right)\right)}}+\prod_{j=1}^{n}\left(1-\left(\gamma_{j}\right)^{2}\right)^{\frac{\omega_{j}\left(1+T\left(\tilde{d}_{j}\right)\right)}{\sum_{j=1}^{n} \omega_{j}\left(1+T\left(\widetilde{d}_{j}\right)\right)}}}}\right\}, \\
& \left\{\frac{\sqrt{2} \prod_{j=1}^{n}\left(\eta_{j}\right)^{\frac{\omega_{j}\left(1+T\left(\tilde{d}_{j}\right)\right)}{\sum_{j=1}^{n} \omega_{j}\left(1+T\left(\tilde{d}_{j}\right)\right)}}}{\sqrt{\prod_{j=1}^{n}\left(2-\left(\eta_{j}\right)^{2}\right)^{\frac{\omega_{j}\left(1+T\left(\tilde{d}_{j}\right)\right)}{\sum_{j=1}^{n} \omega_{j}\left(1+T\left(\tilde{d}_{j}\right)\right)}}+\prod_{j=1}^{n}\left(\eta_{j}\right)^{\frac{2 \omega_{j}\left(1+T\left(\widetilde{d}_{j}\right)\right)}{\sum_{j=1}^{n} \omega_{j}\left(1+T\left(\tilde{d}_{j}\right)\right)}}}}\right\}
\end{aligned}
$$




\subsection{Dual hesitant Pythagorean fuzzy Hamacher power weighted geometric (DHPFHPWG) operator}

$\mathrm{Xu}$ and Yager [44] developed power geometric (PG) operator on the basis of PA operator [43] and geometric mean [45-46], which can be defined as follows:

$$
P G\left(a_{1}, a_{2}, \cdots, a_{n}\right)=\prod_{i=1}^{n}\left(a_{i}\right)^{\left(1+T\left(a_{i}\right)\right)} / \sum_{i=1}^{n}\left(1+T\left(a_{i}\right)\right)
$$

where $T\left(a_{i}\right)=\sum_{\substack{j=1 \\ j \neq i}}^{n} \operatorname{Sup}\left(a_{i}, a_{j}\right)$, and $\operatorname{Sup}(a, b)$ is the support for $a$ from $b$, which satisfies the following three properties:

(1) $\operatorname{Sup}(a, b) \in[0,1]$;

(2) $\operatorname{Sup}(a, b)=\operatorname{Sup}(b, a)$;

(3) $\operatorname{Sup}(a, b) \geqslant \operatorname{Sup}(x, y)$, if $|a-b|<|x-y|$.

In this section, we shall propose the dual hesitant Pythagorean fuzzy Hamacher power weighted geometric (DHPFHPWG) operator based on the power geometric [44] operators and Hamacher operations [36].

Definition 12 Let $\widetilde{d}_{j}(j=1,2, \cdots, n)$ be a collection of DHPFNs, then we define the dual hesitant Pythagorean fuzzy Hamacher power weighted geometric (DHPFHPWG) operator as follows:

$$
\operatorname{DHPFHPWG}\left(\widetilde{d}_{1}, \widetilde{d}_{2}, \cdots, \widetilde{d}_{n}\right)=\bigoplus_{j=1}^{n}\left(\widetilde{d}_{j}\right)=\cup_{\gamma_{j} \in h_{j}, \eta_{j} \in g_{j}}
$$

$$
\left\{\frac{\sqrt{\gamma} \prod_{j=1}^{n}\left(\gamma_{j}\right)^{\frac{\omega_{j}\left(1+T\left(\widetilde{d}_{j}\right)\right)}{\sum_{j=1}^{n} \omega_{j}\left(1+T\left(\widetilde{d}_{j}\right)\right)}}}{\sqrt{\prod_{j=1}^{n}\left(1+(\gamma-1)\left(1-\left(\gamma_{j}\right)^{2}\right)\right)^{\frac{\omega_{j}\left(1+T\left(\tilde{d}_{j}\right)\right)}{\sum_{j=1}^{n} \omega_{j}\left(1+T\left(\widetilde{d}_{j}\right)\right)}}+(\gamma-1) \prod_{j=1}^{n}\left(\gamma_{j}\right)^{\frac{2 \omega_{j}\left(1+T\left(\tilde{d}_{j}\right)\right)}{\sum_{j=1}^{n} \omega_{j}\left(1+T\left(\widetilde{d}_{j}\right)\right)}}}}\right\},
$$

$$
\left.\left\{\sqrt{\frac{\prod_{j=1}^{n}\left(1+(\gamma-1)\left(\eta_{j}\right)^{2}\right)^{\frac{\omega_{j}\left(1+T\left(\tilde{d}_{j}\right)\right)}{\sum_{j=1}^{n} \omega_{j}\left(1+T\left(\tilde{d}_{j}\right)\right)}}-\prod_{j=1}^{n}\left(1-\left(\eta_{j}\right)^{2}\right)^{\frac{\omega_{j}\left(1+T\left(\tilde{d}_{j}\right)\right)}{\sum_{j=1}^{n} \omega_{j}\left(1+T\left(\tilde{d}_{j}\right)\right)}}}{\prod_{j=1}^{n}\left(1+(\gamma-1)\left(\eta_{j}\right)^{2}\right)^{\frac{\omega_{j}\left(1+T\left(\tilde{d}_{j}\right)\right)}{\sum_{j=1}^{n} \omega_{j}\left(1+T\left(\widetilde{d}_{j}\right)\right)}}+(\gamma-1) \prod_{j=1}^{n}\left(1-\left(\eta_{j}\right)^{2}\right)^{\frac{2 \omega_{j}\left(1+T\left(\tilde{d}_{j}\right)\right)}{\sum_{j=1}^{n} \omega_{j}\left(1+T\left(\tilde{d}_{j}\right)\right)}}}}\right\}\right\}
$$


where $\omega=\left(\omega_{1}, \omega_{2}, \cdots, \omega_{n}\right)^{T}$ be the weight vector of $\widetilde{d}_{j}(j=1,2, \cdots, n), \gamma>0$, and

$$
T\left(\widetilde{d}_{j}\right)=\sum_{\substack{i=1 \\ i \neq j}}^{n} \omega_{i} \operatorname{Sup}\left(\widetilde{d}_{j}, \widetilde{d}_{i}\right)
$$

and $\operatorname{Sup}\left(\widetilde{d}_{j}, \widetilde{d}_{i}\right)$ is the support for $\widetilde{d}_{j}$ from $\widetilde{d}_{i}$, with the conditions:

(1) $\operatorname{Sup}\left(\widetilde{d}_{i}, \widetilde{d}_{j}\right) \in[0,1]$;

(2) $\operatorname{Sup}\left(\widetilde{d}_{i}, \widetilde{d}_{j}\right)=\operatorname{Sup}\left(\widetilde{d}_{i}, \widetilde{d}_{j}\right)$;

(3) $\operatorname{Sup}\left(\widetilde{d}_{i}, \widetilde{d}_{j}\right) \geqslant \operatorname{Sup}\left(\widetilde{d}_{s}, \widetilde{d}_{t}\right)$, if dis $\left(\widetilde{d}_{i}, \widetilde{d}_{j}\right) \geqslant \operatorname{dis}\left(\widetilde{d}_{s}, \widetilde{d}_{t}\right)$ where dis is a distance measure.

Now, we can discuss some special cases of the DHPFHPWG operator with respect to the parameter $\gamma$.

- When $\gamma=1$, DHPFHPWG operator reduces to the dual hesitant Pythagorean fuzzy power weighted geometric (DHPFPWG) operator as follows:

$$
\begin{aligned}
& \operatorname{DHPFPWGG}_{\omega}\left(\widetilde{d}_{1}, \widetilde{d}_{2}, \cdots, \widetilde{d}_{n}\right)=\bigotimes_{j=1}^{n}\left(\widetilde{d}_{j}\right)^{\omega_{j}\left(1+T\left(\widetilde{d}_{j}\right)\right) / \sum_{j=1}^{n} \omega_{j}\left(1+T\left(\widetilde{d}_{j}\right)\right)} \\
& =\cup_{\gamma_{j} \in h_{j}, \eta_{j} \in g_{j}}\left\{\left\{\prod_{j=1}^{n}\left(\gamma_{j}\right)^{\omega_{j}\left(1+T\left(\widetilde{d_{j}}\right)\right) / \sum_{j=1}^{n} \omega_{j}\left(1+T\left(\widetilde{d_{j}}\right)\right)}\right\},\right. \\
& \left.\left\{\sqrt{1-\prod_{j=1}^{n}\left(1-\left(\eta_{j}\right)^{2}\right)^{\omega_{j}\left(1+T\left(\widetilde{d}_{j}\right)\right) / \sum_{j=1}^{n} \omega_{j}\left(1+T\left(\widetilde{d}_{j}\right)\right)}}\right\}\right\}
\end{aligned}
$$

- When $\gamma=2$, DHPFHPWG operator reduces to the dual hesitant Pythagorean fuzzy Einstein power weighted geometric (DHPFEPWG) operator as follows:

$$
\begin{gathered}
\operatorname{DHPFEPWG}_{\omega}\left(\widetilde{d}_{1}, \widetilde{d}_{2}, \cdots, \widetilde{d}_{n}\right)=\bigoplus_{j=1}^{n}\left(\widetilde{d}_{j}\right)=\cup_{\gamma_{j} \in h_{j}, \eta_{j} \in g_{j}} \\
\left\{\begin{array}{c}
\sqrt{2} \prod_{j=1}^{n}\left(\gamma_{j}\right)^{\frac{\omega_{j}\left(1+T\left(\tilde{d}_{j}\right)\right)}{\sum_{j=1}^{n} \omega_{j}\left(1+T\left(\widetilde{d}_{j}\right)\right)}} \\
\sqrt{\prod_{j=1}^{n}\left(2-\left(\gamma_{j}\right)^{2}\right)^{\frac{\omega_{j}\left(1+T\left(\tilde{d}_{j}\right)\right)}{\sum_{j=1}^{n} \omega_{j}\left(1+T\left(\tilde{d}_{j}\right)\right)}}+\prod_{j=1}^{n}\left(\gamma_{j}\right)^{\frac{2 \omega_{j}\left(1+T\left(\tilde{d}_{j}\right)\right)}{\sum_{j=1}^{n} \omega_{j}\left(1+T\left(\widetilde{d}_{j}\right)\right)}}}
\end{array},\right.
\end{gathered}
$$




$$
\left\{\sqrt{\frac{\prod_{j=1}^{n}\left(1+\left(\eta_{j}\right)^{2}\right)^{\frac{\omega_{j}\left(1+T\left(\tilde{d}_{j}\right)\right)}{\sum_{j=1}^{n} \omega_{j}\left(1+T\left(\tilde{d}_{j}\right)\right)}}-\prod_{j=1}^{n}\left(1-\left(\eta_{j}\right)^{2}\right)^{\frac{\omega_{j}\left(1+T\left(\tilde{d}_{j}\right)\right)}{\sum_{j=1}^{n} \omega_{j}\left(1+T\left(\tilde{d}_{j}\right)\right)}}}{\prod_{j=1}^{n}\left(1+(\eta)^{2}\right)^{\frac{\omega_{j}\left(1+T\left(\tilde{d}_{j}\right)\right)}{\sum_{j=1}^{n} \omega_{j}\left(1+T\left(\widetilde{d}_{j}\right)\right)}}+\prod_{j=1}^{n}\left(1-\left(\eta_{j}\right)^{2}\right)^{\frac{\omega_{j}\left(1+T\left(\tilde{d}_{j}\right)\right)}{\sum_{j=1}^{n} \omega_{j}\left(1+T\left(\widetilde{d}_{j}\right)\right)}}}}\right\}
$$

\section{An approach to multiple attribute decision making with dual hesitant Pythagorean fuzzy information}

In this section, we shall utilize the dual hesitant aggregation operators to multiple attribute decision making with dual hesitant Pythagorean fuzzy information. Let $A=\left\{A_{1}, A_{2}, \cdots, A_{m}\right\}$ be a discrete set of alternatives, and $G=\left\{G_{1}, G_{2}, \cdots, G_{n}\right\}$ be the state of nature. If the decision makers provide several values for the alternative $A_{i}$ under the state of nature $G_{j}$ with anonymity, these values can be considered as a dual hesitant Pythagorean fuzzy element $\widetilde{d}_{i j}=\left(h_{i j}, g_{i j}\right)$. In the case where two decision makers provide the same value, then the value emerges only once in $\widetilde{d}_{i j}$. Suppose that the decision matrix $\widetilde{D}=\left(\widetilde{d}_{i j}\right)_{m \times n}$ is the dual hesitant Pythagorean fuzzy decision matrix, where $\widetilde{d}_{i j}(i=1,2, \cdots, m, j=1,2, \cdots, n)$ are in the form of DHPFNs.

In the following, we apply the DHPFHWA (or DHPFHWG) operator to the MADM problems for potential evaluation of emerging technology commercialization with dual hesitant Pythagorean fuzzy information.

Step 1 We utilize the decision information given in matrix $\widetilde{D}$, and the DHPFHWA operator

$$
\left.\begin{array}{l}
\widetilde{d}_{i}=\operatorname{DHPFHWA}\left(\widetilde{d}_{i 1}, \tilde{d}_{i 2}, \cdots, \tilde{d}_{i n}\right) \\
=\bigoplus_{j=1}^{n}\left(\omega_{j} \widetilde{d}_{i j}\right) \\
\left.=\cup_{\gamma_{i j} \in h_{i j}, \eta_{i j} \in h_{i j}}\left\{\frac{\left\{\sqrt{\frac{\prod_{j=1}^{n}\left(1+(\gamma-1)\left(\gamma_{i j}\right)^{2}\right)^{\omega_{j}}-\prod_{j=1}^{n}\left(1-\left(\gamma_{i j}\right)^{2}\right)^{\omega_{j}}}{\prod_{j=1}^{n}\left(1+(\gamma-1)\left(\gamma_{i j}\right)^{2}\right)^{\omega_{j}}+(\gamma-1) \prod_{j=1}^{n}\left(1-\left(\gamma_{i j}\right)^{2}\right)^{\omega_{j}}}}\right\},}{\sqrt{\prod_{j=1}^{n}\left(1+(\gamma-1)\left(1-\left(\eta_{i j}\right)^{2}\right)\right)^{\omega_{j}}+(\gamma-1) \prod_{j=1}^{n}\left(\eta_{i j}\right)^{2 \omega_{j}}}}\right\}\right\}
\end{array}\right\}
$$


Or the dual hesitant Pythagorean fuzzy weighted geometric (DHPFHWG) operator:

$$
\begin{aligned}
& \tilde{d}_{i}=\operatorname{DHPFHWG}\left(\widetilde{d}_{i 1}, \widetilde{d}_{i 2}, \cdots, \widetilde{d}_{i n}\right) \\
& =\stackrel{\otimes}{j=1}_{n}^{n}\left(\widetilde{d}_{i j}\right)^{\omega_{j}} \\
& =\cup_{\gamma_{i j} \in h_{i j}, \eta_{i j} \in h_{i j}}\left\{\left\{\frac{\sqrt{\gamma} \prod_{j=1}^{n}\left(\gamma_{i j}\right)^{\omega_{j}}}{\sqrt{\prod_{j=1}^{n}\left(1+(\gamma-1)\left(1-\left(\gamma_{i j}\right)^{2}\right)\right)^{\omega_{j}}+(\gamma-1) \prod_{j=1}^{n}\left(\gamma_{i j}\right)^{2 \omega_{j}}}}\right\},\right. \\
& \left.\left\{\sqrt{\frac{\prod_{j=1}^{n}\left(1+(\gamma-1)\left(\eta_{i j}\right)^{2}\right)^{\omega_{j}}-\prod_{j=1}^{n}\left(1-\left(\eta_{i j}\right)^{2}\right)^{\omega_{j}}}{\prod_{j=1}^{n}\left(1+(\gamma-1)\left(\eta_{i j}\right)^{2}\right)^{\omega_{j}}+(\gamma-1) \prod_{j=1}^{n}\left(1-\left(\eta_{i j}\right)^{2}\right)^{\omega_{j}}}}\right\}\right\}
\end{aligned}
$$

to derive the overall preference values $\widetilde{d}_{i}(i=1,2, \cdots, m)$ of the alternative $A_{i}$.

Step 2 Calculate the scores $S\left(\widetilde{d}_{i}\right)(i=1,2, \cdots, m)$ of the overall dual hesitant Pythagorean fuzzy preference values $\widetilde{d}_{i}(i=1,2, \cdots, m)$. If there is no difference between two scores $S\left(\widetilde{d}_{i}\right)$ and $S\left(\widetilde{d}_{j}\right)$, then we need to calculate the accuracy degrees $S\left(\widetilde{p}_{i}\right)$ and $S\left(\widetilde{p}_{i}\right)$ of the collective overall preference values $\widetilde{d}_{i}$ and $\widetilde{d}_{j}$, respectively, and then rank the alternatives $A_{i}$ and $A_{j}$ in accordance with the accuracy degrees $p\left(\widetilde{d}_{i}\right)$ and $p\left(\widetilde{d}_{i}\right)$.

Step 3 Rank all the alternatives $A_{i}(i=1,2, \cdots, m)$ and select the best one(s) in accordance with the scores $S\left(\widetilde{d_{i}}\right)(i=1,2, \cdots, m)$.

Step 3 End.

\section{Numerical example}

Thus, in this section we shall present a numerical example for supplier selection in supply chain management with dual hesitant Pythagorean fuzzy information in order to illustrate the method proposed in this paper. Let us suppose there is a problem to deal with the supplier selection in supply chain management which is classical multiple attribute decision making problems. There are five prospect suppliers $A_{i}(i=1,2,3,4,5)$ for four attributes $G_{j}(j=1,2,3,4)$. The four attributes include product quality $\left(G_{1}\right)$, service $\left(G_{2}\right)$, delivery $\left(G_{3}\right)$ and price $\left(G_{4}\right)$, respectively. In order to avoid influence each 
other, the decision makers are required to evaluate the five suppliers $A_{i}(i=1,2,3,4,5)$ under the above four attributes in anonymity and the decision matrix $\widetilde{D}=\left(\widetilde{d}_{i j}\right)_{5 \times 4}$ is presented in Tab. 1 , where $\widetilde{d}_{i j}(i=1,2,3,4,5, j=1,2,3,4)$ are in the form of DHPFNs.

Table 1: Dual hesitant Pythagorean fuzzy decision matrix

\begin{tabular}{lllll}
\hline & $G_{1}$ & $G_{2}$ & $G_{3}$ & $G_{4}$ \\
\hline A1 & $\{\{0.3,0.4\},\{0.6\}\}$ & $\{\{0.4,0.5\},\{0.3,0.4\}\}$ & $\{\{0.2,0.3\},\{0.7\}\}$ & $\{\{0.4,0.5\},\{0.5\}\}$ \\
A2 & $\{\{0.6\},\{0.4\}\}$ & $\{\{0.2,0.4,0.5\},\{0.4\}\}$ & $\{\{0.2\},\{0.6,0.7,0.8\}\}$ & $\{\{0.5\},\{0.4,0.5\}\}$ \\
A3 & $\{\{0.5,0.7\},\{0.2\}\}$ & $\{\{0.2\},\{0.7,0.8\}\}$ & $\{\{0.2,0.3,0.4\},\{0.6\}\}$ & $\{\{0.5,0.6,0.7\},\{0.3\}\}$ \\
A4 & $\{\{0.7\},\{0.3\}\}$ & $\{\{0.6,0.7,0.8\},\{0.2\}\}$ & $\{\{0.1,0.2\},\{0.3\}\}$ & $\{\{0.1\},\{0.6,0.7,0.8\}\}$ \\
A5 & $\{\{0.6,0.7\},\{0.2\}\}$ & $\{\{0.2,0.3,0.4\},\{0.5\}\}$ & $\{\{0.4,0.5\},\{0.2\}\}$ & $\{\{0.2,0.3,0.4\},\{0.5\}\}$ \\
\hline
\end{tabular}

The information about the attribute weights is known as follows: $\omega=$ $(0.20,0.15,0.35,0.30)$. In the following, we utilize the approach developed for supplier selection in supply chain management with dual hesitant Pythagorean fuzzy information.

Step 1 We utilize the decision information given in matrix $\widetilde{D}$, and the DHPFHWA operator to obtain the overall preference values $\widetilde{d}_{i}$ of the supplier in supply chain management $A_{i}(i=1,2,3,4,5)$. Take alternative $A_{i}$ for an example (here, we take $\gamma=3$ ), we have

$$
\begin{aligned}
& \widetilde{d}_{1}=\operatorname{DHPFHWA}_{\omega}\left(\widetilde{d}_{11}, \widetilde{d}_{12}, \widetilde{d}_{13}, \widetilde{d}_{14}\right) \\
& =\bigoplus_{j=1}^{4}\left(\omega_{j} \widetilde{d}_{1 j}\right) \\
& =\cup_{\gamma_{1 j} \in h_{1 j}, \eta_{1 j} \in h_{1 j}}\left\{\sqrt{\frac{\prod_{j=1}^{4}\left(1+(\gamma-1)\left(\gamma_{1 j}\right)^{2}\right)^{\omega_{j}}-\prod_{j=1}^{4}\left(1-\left(\gamma_{1 j}\right)^{2}\right)^{\omega_{j}}}{\prod_{j=1}^{4}\left(1+(\gamma-1)\left(\gamma_{1 j}\right)^{2}\right)^{\omega_{j}}+(\gamma-1) \prod_{j=1}^{4}\left(1-\left(\gamma_{1 j}\right)^{2}\right)^{\omega_{j}}}}\right\}, \\
& \left\{\frac{\sqrt{\gamma} \prod_{j=1}^{4}\left(\eta_{1 j}\right)^{\omega_{j}}}{\sqrt{\prod_{j=1}^{4}\left(1+(\gamma-1)\left(1-\left(\eta_{1 j}\right)^{2}\right)\right)^{\omega_{j}}+(\gamma-1) \prod_{j=1}^{4}\left(\eta_{1 j}\right)^{2 \omega_{j}}}}\right\} \\
& =\{\{\{0.3,0.4\},\{0.5\}\},\{\{0.4,0.5\},\{0.3,0.4\}\},\{\{0.2,0.3\},\{0.5\}\},\{\{0.4,0.5\},\{0.5\}\}\} \\
& =\{\{0.3005,0.3146,0.3281,0.3328,0.3333,0.3412,0.3457,0.3461,0.3582,0.3586 \text {, } \\
& 0.3630,0.3703,0.3707,0.3749,0.3866,0.3979\},\{0.3097,0.3416\}\}
\end{aligned}
$$


Step 2 Calculate the scores $s\left(\widetilde{d}_{i}\right)(i=1,2,3,4,5)$ of the overall dual hesitant Pythagorean fuzzy preference values $\widetilde{d}_{i}(i=1,2,3,4,5)$ :

$$
\begin{aligned}
& s\left(\tilde{d}_{1}\right)=0.3828, s\left(\tilde{d}_{2}\right)=0.4552, s\left(\widetilde{d}_{3}\right)=0.5008 \\
& s\left(\widetilde{d}_{4}\right)=0.4774, s\left(\widetilde{d}_{5}\right)=0.6171
\end{aligned}
$$

Step 3 Rank all the suppliers $A_{i}(i=1,2,3,4,5)$ in accordance with the scores $s\left(\widetilde{d}_{i}\right)(i=1,2,3,4,5)$ of the overall dual hesitant Pythagorean fuzzy numbers: $A_{5} \succ$ $A_{3} \succ A_{4} \succ A_{2} \succ A_{1}$, and thus the most desirable supplier is $A_{5}$.

Based on the DHPFHWG operator, then, in order to select the most desirable supplier, we can develop an approach to multiple attribute decision making problems with dual hesitant Pythagorean fuzzy information, which can be described as following:

Step 1' Aggregate all dual hesitant Pythagorean fuzzy value $\widetilde{h}_{i j}(j=1,2,3,4)$ by using the dual hesitant Pythagorean fuzzy weighted geometric (DHPFHWG) operator to derive the overall dual hesitant Pythagorean fuzzy values $\widetilde{d}_{i}(i=1,2, \cdots, 5)$ of the supplier $A_{i}$. Take supplier $A_{1}$ for an example (here, we take $\gamma=3$ ), we have

$$
\begin{aligned}
& \widetilde{d}_{1}=\operatorname{DHPFHWG}_{\omega}\left(\widetilde{d}_{11}, \widetilde{d}_{12}, \widetilde{d}_{13}, \widetilde{d}_{14}\right) \\
& =\otimes_{j=1}^{4}\left(\widetilde{d}_{1 j}\right)^{\omega_{j}} \\
& =\cup_{\gamma_{1 j} \in h_{1 j}, \eta_{1 j} \in h_{1 j}}\left\{\left\{\frac{\sqrt{\gamma} \prod_{j=1}^{4}\left(\gamma_{1 j}\right)^{\omega_{j}}}{\sqrt{\prod_{j=1}^{4}\left(1+(\gamma-1)\left(1-\left(\gamma_{1 j}\right)^{2}\right)\right)^{\omega_{j}}+(\gamma-1) \prod_{j=1}^{4}\left(\gamma_{1 j}\right)^{2 \omega_{j}}}}\right\},\right. \\
& \left.\left\{\sqrt{\frac{\prod_{j=1}^{4}\left(1+(\gamma-1)\left(\eta_{1 j}\right)^{2}\right)^{\omega_{j}}-\prod_{j=1}^{4}\left(1-\left(\eta_{1 j}\right)^{2}\right)^{\omega_{j}}}{\prod_{j=1}^{4}\left(1+(\gamma-1)\left(\eta_{1 j}\right)^{2}\right)^{\omega_{j}}+(\gamma-1) \prod_{j=1}^{4}\left(1-\left(\eta_{1 j}\right)^{2}\right)^{\omega_{j}}}}\right\}\right\} \\
& =\{\{\{0.3,0.4\},\{0.5\}\},\{\{0.4,0.5\},\{0.3,0.4\}\},\{\{0.2,0.3\},\{0.5)\},\{\{0.4,0.5\},\{0.5\}\}\} \\
& =\{\{0.2794,0.2863,0.2934,0.3006,0.3053,0.3128,0.3204,0.3275,0.3282,0.3354 \text {, } \\
& 0.3435,0.3518,0.3572,0.3657,0.3743,0.3832\},\{0.5913,0.6031\}\}
\end{aligned}
$$


Step 2' Calculate the scores $s\left(\widetilde{d}_{i}\right)(i=1,2,3,4,5)$ of the overall dual hesitant Pythagorean fuzzy values $\widetilde{d}_{i}(i=1,2,3,4,5)$ of the supplier $A_{i}$ :

$$
\begin{aligned}
& s\left(\widetilde{d}_{1}\right)=0.4862, s\left(\widetilde{d}_{2}\right)=0.4084, s\left(\widetilde{d}_{3}\right)=0.4076 \\
& s\left(\widetilde{d}_{4}\right)=0.4941, s\left(\widetilde{d}_{5}\right)=0.5502
\end{aligned}
$$

Step 3' Rank all the suppliers in supply chain management $A_{i}(i=1,2,3,4,5)$ in accordance with the scores $s\left(\widetilde{d}_{i}\right)(i=1,2,3,4,5)$ of the overall dual hesitant Pythagorean fuzzy values $\widetilde{d_{i}}(i=1,2, \cdots, 5)$ by using definition 5 : $A_{5} \succ A_{4} \succ A_{1} \succ A_{2} \succ A_{3}$ and thus the most desirable supplier in supply chain management is $A_{5}$.

From the above analysis, it is easily seen that although the overall rating values of the alternatives are slightly different by using two operators respectively. However, the most desirable supplier in supply chain management is $A_{5}$.

\section{Conclusion}

In this paper, we investigate the multiple attribute decision making (MADM) problem based on the Hamacher aggregation operators with dual Pythagorean hesitant fuzzy information. Then, motivated by the ideal of Hamacher operation, we have developed some Hamacher aggregation operators for aggregating dual hesitant Pythagorean fuzzy information: dual hesitant Pythagorean fuzzy Hamacher weighted average (DHPFHWA) operator, dual hesitant Pythagorean fuzzy Hamacher weighted geometric (DHPFHWG) operator, dual hesitant Pythagorean fuzzy Hamacher ordered weighted average (DHPFHOWA) operator, dual hesitant Pythagorean fuzzy Hamacher ordered weighted geometric (DHPFHOWG) operator, dual hesitant Pythagorean fuzzy Hamacher hybrid average (DHPFHHA) operator and dual hesitant Pythagorean fuzzy Hamacher hybrid geometric (DHPFHHG) operator. The prominent characteristic of these proposed operators are studied. Then, we have utilized these operators to develop some approaches to solve the dual hesitant Pythagorean fuzzy multiple attribute decision making problems. Finally, a practical example for supplier selection in supply chain management is given to verify the developed approach and to demonstrate its practicality and effectiveness. In the future, we shall continue working in the extension and application of the developed operators to other domains and uncertain environments [47-66]. 


\section{References}

[1] K. Atanassov: Intuitionistic fuzzy sets. Fuzzy Sets and Systems, 20 (1986), 8796.

[2] K. Atanassov: More on intuitionistic fuzzy sets. Fuzzy Sets and Systems, $\mathbf{3 3}$ (1989), 37-46.

[3] L.A. ZADEH: Fuzzy sets. Information and Control, 8 (1965), 338-356.

[4] Z.S. XU: Intuitionistic fuzzy aggregation operators. IEEE Trans. on Fuzzy Systems, 15(6), (2007), 1179-1187.

[5] Z.S. XU and R.R. YAGER: Some geometric aggregation operators based on intuitionistic fuzzy sets. Int. J. of General System, 35 (2006), 417-433.

[6] Z.S. XU and R.R. YAGER: Dynamic intuitionistic fuzzy multi-attribute decision making. Int. J. of Approximate Reasoning, 48(1), (2008), 246-262.

[7] G.W. WeI: Some geometric aggregation functions and their application to dynamic multiple attribute decision making in intuitionistic fuzzy setting. Int. J. of Uncertainty, Fuzziness and Knowledge- Based Systems, 17(2), (2009), 179-196.

[8] G.W. WEI: Some induced geometric aggregation operators with intuitionistic fuzzy information and their application to group decision making. Applied Soft Computing, 10(2), (2010), 423-431.

[9] G.W. WeI and X.F. ZhaO: Some induced correlated aggregating operators with intuitionistic fuzzy information and their application to multiple attribute group decision making. Expert Systems with Applications, 39(2), (2012), 2026-2034.

[10] D.J. YU, Y.Y. WU and T. LU: Intuitionistic fuzzy prioritized operators and their application in group decision making. Knowledge-Based Systems, 30 (2012), 5766.

[11] Z.S. XU: Approaches to multiple attribute group decision making based on intuitionistic fuzzy power aggregation operators.; Knowledge-Based Systems, 24(6), (2011), 749-760.

[12] Z.S. XU and Q. CHEN: A multi-criteria decision making procedure based on intuitionistic fuzzy bonferroni means. J. of Systems Science and Systems Engineering, 20(2), (2011), 217-228.

[13] Z.S. XU and M.M. XIA: Induced generalized intuitionistic fuzzy operators. Knowledge-Based Systems, 24(2), (2011), 197-209.

[14] DeJIAn Yu: Intuitionistic fuzzy geometric Heronian mean aggregation operators. Applied Soft Computing, 13(2), (2013), 1235-1246. 
[15] JiAn-QIAng WAng, Rong-Rong NiE, Hong-Yu ZHANG and XiaO-Hong CHEN: Intuitionistic fuzzy multi-criteria decision-making method based on evidential reasoning. Applied Soft Computing, 13(4), (2013), 1823-1831.

[16] G.W. WEI: Approaches to interval intuitionistic trapezoidal fuzzy multiple attribute decision making with incomplete weight information. Int. J. of Fuzzy Systems, 17(3), (2015), 484-489.

[17] TAMAlika Chaira: Enhancement of medical images in an Atanassov's't intuitionistic fuzzy domain using an alternative intuitionistic fuzzy generator with application to image segmentation. J. of Intelligent and Fuzzy Systems, 27(3), (2014), 1347-1359.

[18] Sujit Kumar De and Shib SAnkar SAnA: A multi-periods productioninventory model with capacity constraints for multi-manufacturers - A global optimality in intuitionistic fuzzy environment. Applied Mathematics and Computation, 242 (2014), 825-841.

[19] Feifei Jin, LidAn Pei, HuAyou Chen and Ligang Zhou: Interval-valued intuitionistic fuzzy continuous weighted entropy and its application to multi-criteria fuzzy group decision making. Knowledge-Based Systems, 59 (2014), 132-141.

[20] Rajkumar Verma and Bhu Dev Sharma: A new measure of inaccuracy with its application to multi-criteria decision making under intuitionistic fuzzy environment. J. of Intelligent and Fuzzy Systems, 27(4), (2014), 1811-1824.

[21] TING-YU CHEN: The inclusion-based TOPSIS method with interval-valued intuitionistic fuzzy sets for multiple criteria group decision making. Applied Soft Computing, 26 (2015), 57-73.

[22] IRFAN DELI and NAIM ÇAGMAN: Intuitionistic fuzzy parameterized soft set theory and its decision making. Applied Soft Computing, 28 (2015), 109-113.

[23] G.W. WEI, H.J. WANG and R. LIN: Application of correlation coefficient to interval-valued intuitionistic fuzzy multiple attribute decision making with incomplete weight information. Knowledge and Information Systems, 26(2), (2011), 337349.

[24] Shouzhen ZENG and YAO XIAO: TOPSIS method for intuitionistic fuzzy multiple-criteria decision making and its application to investment selection. $K y$ bernetes, 45(2), (2016), 282-296.

[25] Ali Shakiba, Mohammad Reza Hooshmandasl, Bijan Davvaz and Seyed Abolfazl Shahzadeh Fazeli: An intuitionistic fuzzy approach to Sapproximation spaces. J. of Intelligent and Fuzzy Systems, 30(6), (2016), 33853397. 
[26] Feng Shen, Jiuping Xu and Zeshui Xu: An outranking sorting method for multi-criteria group decision making using intuitionistic fuzzy sets. Information Sciences, 334 (2016), 338-353.

[27] X.F. ZHAO and G.W. WEI: Some intuitionistic fuzzy Einstein hybrid aggregation operators and their application to multiple attribute decision making. KnowledgeBased Systems, 37 (2013), 472-479.

[28] Y. TANG, L.L. WEN and G.W. WEI: Approaches to multiple attribute group decision making based on the generalized Dice similarity measures with intuitionistic fuzzy information. Int. J. of Knowledge-Based and Intelligent Engineering Systems, 21(2), (2017), 85-95.

[29] R.R. YAGER: Pythagorean fuzzy subsets. In: Proc. of the Joint IFSA World Congress and NAFIPS Annual Meeting, Edmonton, Canada, (2013), 57-61.

[30] R.R. YAGER: Pythagorean membership grades in multicriteria decision making. IEEE Trans. on Fuzzy Systems, 22 (2014), 958-965.

[31] X.L.ZHANG and Z.S. XU: Extension of TOPSIS to multiple criteria decision making with Pythagorean fuzzy sets. Int. J. Intellingent Systems, 29 (2014) 1061-1078.

[32] X. PEng and Y.YAng: Some results for Pythagorean fuzzy sets. Int. J. Intelligent Systems, 30 (2015), 1133-1160.

[33] G. BELIAKOV and S. JAMES: Averaging aggregation functions for preferences expressed as Pythagorean membership grades and fuzzy orthopairs. IEEE Int. Conf. on Fuzzy Systems, (2014), 298-305.

[34] M.Z. REFORMAT and R.R. YAGER: Suggesting recommendations using Pythagorean fuzzy sets illustrated using Netflix Movie Data. in Proc. Int. Conf. on Information Processing and Management of Uncertainty in Knowledge-Based Systems, Mompelier, France, 1 (2014), 546-556.

[35] Bin Zhu, Zeshui XU and Meimei XiA: Dual hesitant fuzzy sets. J. of Applied Mathematics, (2012), Article ID 879629, 13 pages. http://www.hindawi.com/journals/jam/2012/879629/.

[36] G. Beliakov, A. Pradera and T. CAlvo: Aggregation Functions: A Guide For Practitioners. Heidelberg, Germany, Springer, 2007.

[37] H. HAMACHAR: Über logische Vernupfungen unscharfer Aussagen und deren Zugehörige Bewertungsfunctionen. In Trappl, Klir, Riccardi (Eds.), Progress in Cybernatics and systems research, 3 Hemisphere, Washington DC, 1978, 276-288.

[38] G. DeschriJver, C. Cornelisand E.E. Kerre: On the representation of intuitionistic fuzzy t-norms and t-conorms. IEEE Trans. on Fuzzy Systems, 12 (2004), 45-61. 
[39] S. RoYchowdhurYand B.H. WANG: On generalized Hamacher families of triangular operators. Int. J. of Approximate Reasoning, 19 (1998), 419-439.

[40] G. DeschriJverand E.E KerRe: A generalization of operators on intuitionistic fuzzy sets using triangular norms and conorms. Notes on Intuitionistic Fuzzy Sets, 8 (2002), 19-27.

[41] Liyong Zhou, Xiaofei Zhaoand Guiwu Wei: Hesitant fuzzy Hamacher aggregation operators and their application to multiple attribute decision making. $J$. of Intelligent and Fuzzy Systems, 26(6), (2014), 2689-2699.

[42] PEIDE LIU: Some Hamacher aggregation operators based on the interval-valued intuitionistic fuzzy numbers and their application to group decision making. IEEE Trans. Fuzzy Systems, 22(1), (2014), 83-97.

[43] R.R. YAGER: The power average operator. IEEE Trans. on Systems, Man, and Cybernetics-Part A, 31(6), (2001), 724-731.

[44] Z. XU and R.R. YAGER: Power-geometric operators and their use in group decision making. IEEE Trans. on Fuzzy Systems, 18(1), (2010), 94-105.

[45] F. Chiclana, F. Herrera and E. Herrera-Viedma: The ordered weighted geometric operator: Properties and application. In: Proc. of 8th Int. Conf. on Information Processing and Management of Uncertainty in Knowledge-based Systems, Madrid, (2000), 985-991.

[46] Z.S. XU and Q.L. DA: An overview of operators for aggregating information. Int. J. of Intelligent System, 18 (2003), 953-969.

[47] J. YE: Multicriteria decision-making method using the Dice similarity measure between expected intervals of trapezoidal fuzzy numbers. J. of Decision Systems, 21(4), (2012), 307-317.

[48] J. YE: Vector similarity measures of simplified neutrosophic sets and their application in multicriteria decision making. Int. J. of Fuzzy Systems, 16(2), (2014), 204-211.

[49] G.W. WeI: Picture fuzzy cross-entropy for multiple attribute decision making problems. J. of Business Economics and Management, 17(4), (2016), 491-502.

[50] G.W. WEI: Picture fuzzy aggregation operators and their application to multiple attribute decision making. J. of Intelligent and Fuzzy Systems, 33(2), (2017), 713724.

[51] G.W. WEI: Picture 2-tuple linguistic Bonferroni mean operators and their application to multiple attribute decision making. Int. J. of Fuzzy System, 19(4), (2017), 997-1010. 
[52] G.W. WEI: Interval-valued dual hesitant fuzzy uncertain linguistic aggregation operators in multiple attribute decision making. J. of Intelligent and Fuzzy Systems, 33(3), (2017), 1881-1893.

[53] G.W. Wei, F.E. Alsand, T. Hayat and A. Alsaedi: Hesitant fuzzy linguistic arithmetic aggregation operators in multiple attribute decision making. Iranian J. of Fuzzy Systems, 13(4), (2016), 1-16.

[54] TING-YU CHEN: The inclusion-based TOPSIS method with interval-valued intuitionistic fuzzy sets for multiple criteria group decision making. Applied Soft Computing, 26 (2015), 57-73.

[55] G.W. Wei, F.E. AlsaAdi, T. Hayat and A. Alsaedi: Hesitant bipolar fuzzy aggregation operators in multiple attribute decision making. J. of Intelligent and Fuzzy Systems, 33(2), (2017), 1119-1128.

[56] Z.S. XU and R.R. YAGER: Some geometric aggregation operators based on intuitionistic fuzzy sets. Int. J. of General Systems, 35 (2006), 417-433.

[57] Z.S. XU: Choquet integrals of weighted intuitionistic fuzzy information. Information Sciences, 180 (2010), 726-736.

[58] M. LU and G.W. WEI: Models for multiple attribute decision making with dual hesitant fuzzy uncertain linguistic information. Int. J. of Knowledge-Based and Intelligent Engineering Systems, 20(4), (2016), 217-227.

[59] M. Lu, G.W. Wei, F.E. Alsandi, T. Hayat and A. Alsaedi: Hesitant Pythagorean fuzzy Hamacher aggregation operators and their application to multiple attribute decision making. J. of Intelligent and Fuzzy Systems, 33(2), (2017), 1105-1117.

[60] J.M. Merigó and A.M. GiL-LAFuEnTE: Induced 2-tuple linguistic generalized aggregation operators and their application in decision-making. Information Sciences, 236(1), (2013), 1-16.

[61] G.W. Wei, F.E. Alsandi, T. Hayat and A. Alsaedi: A linear assignment method for multiple criteria decision analysis with hesitant fuzzy sets based on fuzzy measure. Int. J. of Fuzzy Systems, 19(3), (2017), 607-614.

[62] G.W. WEI, X.R. XU and D.X. DENG: Interval-valued dual hesitant fuzzy linguistic geometric aggregation operators in multiple attribute decision making. Int. J. of Knowledge-Based and Intelligent Engineering Systems, 20(4), (2016), 189-196.

[63] G.W. WEI and J.M. WANG: A comparative study of robust efficiency analysis and data envelopment analysis with imprecise data. Expert Systems with Applications, 81 (2017), 28-38. 
[64] G.W. WEI: Interval valued hesitant fuzzy uncertain linguistic aggregation operators in multiple attribute decision making. Int. J. of Machine Learning and Cybernetics, 7(6), (2016), 1093-1114.

[65] G.W. Wei, M. Lu, F.E. AlsaAdi, T. Hayat and A. Alsaedi: Pythagorean 2tuple linguistic aggregation operators in multiple attribute decision making. J. of Intelligent and Fuzzy Systems, 33(2), (2017), 1129-1142.

[66] M. Lu, G.W. Wei, F.E. AlsaAdi, T. Hayat and A. Alsaedi: Bipolar 2-tuple linguistic aggregation operators in multiple attribute decision making. J. of Intelligent and Fuzzy Systems, 33(2), (2017), 1197-1207. 